\title{
Global existence in reaction-diffusion systems with mass control under relaxed assumptions merely referring to cross-absorptive effects
}

\author{
JOHANNES LANKEIT(D AND MICHAEL WINKLER
}

Abstract. We introduce a generalized concept of solutions for reaction-diffusion systems and prove their global existence. The only restriction on the reaction function beyond regularity, quasipositivity and mass control is special in that it merely controls the growth of cross-absorptive terms. The result covers nonlinear diffusion and does not rely on an entropy estimate.

\section{Introduction}

Reaction-diffusion equations arise in various applications in chemistry and biology (cf. [20, Ch. 2]) and form an important class of model problems in the study of systems of parabolic equations (see [24, Ch. 33]). Already at the stage of basic theories of solvability, a major challenge for the analysis of such systems consists in the presence of commonly superlinear source terms. While the possibility of blow up then is apparrent as long as suitably destabilizing reaction mechanisms are admitted (cf. e.g. [13]), even the requirement of dissipation of mass-which is sufficient to yield global existence and boundedness in the corresponding ODE systems-cannot preclude its occurrence, as impressively demonstrated by the counterexamples in [22]. Global classical solutions have, accordingly, been searched for and found under certain restrictive conditions.

In the context of boundary value problems for systems of the general form

$$
\partial_{t} u_{i}=d_{i} \Delta u_{i}^{m_{i}}+f_{i}\left(u_{1}, \ldots, u_{N}\right), \quad i \in\{1, \ldots, N\},
$$

in the linear diffusion case when $m_{1}=\ldots=m_{N}=1$ such results on global smooth solvability cover settings where boundedness of the first among two components is a priori known (from a sign of $f_{1}, \mathrm{cf}$. [18]), where the diffusion coefficients are close to each other [2,5], or where sources exhibit subquadratic growth [4], and recently ideas of [12] have successfully been extended to show global solvability for quadratic or slightly superquadratic reaction functions $[3,9,26]$.

Mathematics Subject Classification: 35K57, 35D99, 35K59

Keywords: Reaction-diffusion equation, Renormalized solution, Global existence. 
Another line of investigations pursues solutions in a weaker sense. Weak solutions can be constructed if $L^{1}$-bounds for the reaction terms $f_{i}(u)$ are known [19], or if the reaction functions are at most quadratic [21]. For nonlinear diffusion of porous medium type, the existence of weak solutions to (1.4) with Dirichlet boundary data is shown in [15] under the assumptions that

$$
\left\{\begin{array}{lc}
f_{i} \in W_{l o c}^{1, \infty}\left([0, \infty)^{N}\right) \quad \text { is such that } & \\
f_{i}\left(s_{1}, \ldots s_{i-1}, 0, s_{i+1}, \ldots, s_{N}\right) \geq 0 & \text { for all }\left(s_{1}, \ldots, s_{N}\right) \in[0, \infty)^{N} \\
& \text { and } i \in\{1, \ldots, N\},
\end{array}\right.
$$

that with some $K \geq 0$ and $a \in(0, \infty)^{N}$ we have

$$
\begin{array}{cc}
\sum_{i=1}^{N} a_{i} f_{i}\left(s_{1}, \ldots, s_{N}\right) \leq K \cdot\left(\sum_{i=1}^{N} a_{i} s_{i}+1\right) \quad \text { for all }\left(s_{1}, \ldots, s_{N}\right) \in[0, \infty)^{N} \\
\text { and } i \in\{1, \ldots, N\}
\end{array}
$$

and that either a priori $L^{1}$-bounds for the reaction terms are known, or $f_{i}\left(s_{1}, \ldots, s_{N}\right) \geq$ $-C\left(\sum_{j=1}^{N} s_{j}^{\beta_{j}}+1\right)$ with $\beta_{i}<m_{i}+1$ for all $i \in\{1, \ldots, N\}$. An analogous result has been achieved for the corresponding Neumann problem in [14] with a different proof and less restrictive conditions on the initial data, and in the cases when additionally all $m_{i}$ are sufficiently large compared to $\max _{i} \beta_{i}$, higher regularity and convergence of solutions were shown in [8].

The concept of renormalized solutions, that is, the idea that not $u$ itself, but a transformed quantity $\rho(u)$ solves (a weak form) of the equation, makes it possible to bypass even further restrictions on the form of the system. This concept has been successfully introduced for the Boltzmann equation by DiPerna and Lions [7] and was employed for reaction diffusion equations with quadratic reaction functions and linear diffusion in [6]. The apparently most far-reaching application of this idea to reactiondiffusion systems (with linear diffusion) can be found in [10], where essentially no growth restriction on the $f_{i}$ is needed, but where the reaction function is supposed to obey a certain entropy condition. The term in the definition of solutions for whose treatment this entropy condition is essential arises from the choice of renormalization functions $\xi:[0, \infty)^{N} \rightarrow \mathbb{R}$ with compactly supported $D \xi$, which in particular depend on all solution components simultaneously.

Main results. In the present manuscript, we intend to introduce an approach by which it becomes possible to avoid any requirement of the latter type, and it turns out that this can in fact be achieved by resorting to separate renormalization functions for each component $u_{i}$. Thereby, our main result, as stated in Theorem 1.1 below, partially answers the open problem [10, p.585] to find a similar notion of solution without requiring an entropy condition. 
Specifically, we shall be concerned with the Neumann problem

$$
\begin{cases}\partial_{t} u_{i}=d_{i} \Delta u_{i}^{m_{i}}+f_{i}\left(u_{1}, \ldots, u_{N}\right), & x \in \Omega, t>0, i \in\{1, \ldots, N\}, \\ \partial_{\nu} u_{i}^{m_{i}}=0, & x \in \partial \Omega, t>0, i \in\{1, \ldots, N\}, \\ u_{i}(x, 0)=u_{0 i}(x), & x \in \Omega, i \in\{1, \ldots, N\},\end{cases}
$$

under the assumptions that (1.2) and (1.3) hold, and that

$$
\left\{\begin{array}{l}
f_{i}\left(s_{1}, . ., s_{N}\right) \geq-\phi_{i}\left(s_{i}\right) \cdot\left(\sum_{\substack{j \in\{1, \ldots, N\} \\
j \neq i}} s_{j}^{\beta_{j}}+1\right) \text { for all }\left(s_{1}, \ldots, s_{N}\right) \in[0, \infty)^{N} \\
\text { and } i \in\{1, \ldots, N\}, C^{1}([0, \infty)) \text { such that } \phi_{i}^{\prime}>0 \text { on }(0, \infty) \\
\text { with some nonnegative } \phi_{i} \in C^{1}(1.5) \\
\text { for } i \in\{1, \ldots, N\}, \text { and some } \beta_{i}>0 \text { such that } \beta_{i}<m_{i}+1 \text { for all } i \in\{1, \ldots, N\} .
\end{array}\right.
$$

Here we recall that the quasipositivity condition in (1.2) is important in order to avoid negative concentrations, and that (1.3) is a slightly generalized mass dissipation condition, and includes some stoichiometric coefficients $a$. In addition to this, (1.5) signifies a growth condition for the negative parts of the reaction functions, where in the special case of linear diffusion, subquadratic growth is admissible. It is important to note, however, that this restriction only applies to the cross-absorptive effects: For $\left(f_{i}\right)_{-}$, the possible growth with respect to the $i$-th argument remains unrestricted. As for the initial data in (1.4), throughout this paper we shall suppose that

$$
\begin{aligned}
& u_{0 i}, i \in\{1, \ldots, N\}, \text { is a nonnegative function from } L^{r}(\Omega) \text { with some } \\
& \begin{cases}r \geq 1 & \text { if } n=1, \\
r>1 & \text { if } n=2, \\
r \geq \frac{2 n}{n+2} & \text { if } n \geq 3 .\end{cases}
\end{aligned}
$$

Postponing the precise description of the solution concept to be pursued here to Sect. 2, let us introduce our main result obtained in this framework, and give a few examples of its application.

Theorem 1.1. Let $n \geq 1$ and $N \geq 1$ and $\Omega \subset \mathbb{R}^{n}$ a bounded domain with smooth boundary, and suppose that $d_{1}, \ldots, d_{N}$ and $m_{1}, \ldots, m_{N}$ are positive, and that $f_{1}, \ldots, f_{N}$ belong to $W_{\text {loc }}^{1, \infty}\left([0, \infty)^{N}\right)$ and satisfy (1.3), (1.2) and (1.5) with some positive constant $K$. Then given any $u_{01}, \ldots, u_{0 N}$ fulfilling (1.6), one can find nonnegative functions $u_{i} \in L_{l o c}^{m_{i}+1}(\bar{\Omega} \times[0, \infty))$ such that $\left(u_{1}, \ldots, u_{N}\right)$ is a generalized solution of $(1.4)$ in the sense of Definition 2.2.

Remark. (i) The required smoothness of the domain is not the focus of our investigation and could be weakened - in fact, already for the present construction, $C^{2+\alpha}$ regularity for some $\alpha \in(0,1)$ - entering in the construction of classical approximate solutions - would be sufficient.

(ii) Likewise, in order to avoid additional technicalities we do not investigate in detail here whether covering less regular sources, such as e.g. merely continuous $f_{i}$, $i \in\{1, \ldots, N\}$, might be possible at the cost of an additional approximation argument in the context of the regularized versions (3.1) of (1.4) below. 
(iii) An interesting question left open here is how far the regularity requirements in (1.6) could further be relaxed, so as to require integrability of $\left(u_{i 0}\right)_{i \in\{1, \ldots, N\}}$ only, for instance. As will become clear in the proof of Lemma 3.2 below, our currently pursued strategy will crucially rely on (1.6) in order to appropriately control certain initial data appearing in the course of a duality-based reasoning.

Application \#1. A first application of Theorem 1.1 addresses the system

$$
\begin{cases}\partial_{t} u_{i}=d_{i} \Delta u_{i}^{m_{i}}+\left(p_{i}-q_{i}\right) \cdot\left(k_{2} \prod_{j=1}^{N} u_{j}^{q_{j}}-k_{1} \prod_{j=1}^{N} u_{j}^{p_{j}}\right), & x \in \Omega, t>0, i \in\{1, \ldots, N\}, \\ \partial_{\nu} u_{i}^{m_{i}}=0, & x \in \partial \Omega, t>0, i \in\{1, \ldots, N\}, \\ u_{i}(x, 0)=u_{0 i}(x), & x \in \Omega, i \in\{1, \ldots, N\},\end{cases}
$$

which describes a general reversible reaction of the form

$$
p_{1} \mathcal{U}_{1}+p_{2} \mathcal{U}_{2}+\cdots+p_{n} \mathcal{U}_{n} \underset{k_{2}}{\stackrel{k_{1}}{\rightleftharpoons}} q_{1} \mathcal{U}_{1}+q_{2} \mathcal{U}_{2}+\cdots+q_{N} \mathcal{U}_{N}
$$

and for which we obtain the following.

Proposition 1.2. Let $N \geq 2$, and suppose that $k_{1}>0$ and $k_{2}>0$, and that for $i \in\{1, \ldots, N\}, d_{i}>0, m_{i}>0, p_{i} \geq 1$ and $q_{i} \geq 1$ are such that for some $a \in(0, \infty)^{N}$

$$
\sum_{i=1}^{N} a_{i} p_{i}=\sum_{i=1}^{N} a_{i} q_{i}
$$

and that

$$
\sum_{\substack{j \in\{1, \ldots, N\} \\ j \neq i}} \frac{p_{j}}{m_{j}+1}<1 \quad \text { for all } i \in\{1, \ldots, N\} \text { such that } p_{i}>q_{i}
$$

as well as

$$
\sum_{\substack{j \in\{1, \ldots, N\} \\ j \neq i}} \frac{q_{j}}{m_{j}+1}<1 \quad \text { for all } i \in\{1, \ldots, N\} \text { such that } p_{i}<q_{i} .
$$

Then for any choice of $u_{01}, \ldots, u_{0 n}$ complying with (1.6), the problem (1.7) admits a generalized solution in the sense of Definition 2.2.

Proof of Proposition 1.2. Writing $f_{i}\left(s_{1}, \ldots, s_{N}\right):=\left(p_{i}-q_{i}\right) \cdot\left(k_{2} \prod_{j=1}^{N} s_{j}^{q_{j}}-k_{1}\right.$ $\left.\prod_{j=1}^{N} s_{j}^{p_{j}}\right)$ for $i \in\{1, \ldots, N\}$ and $\left(s_{1}, \ldots, s_{N}\right) \in[0, \infty)^{N}$, we see that (1.2) is fulfilled and (1.3) follows since $\sum_{j=1}^{N} a_{i} f_{i} \equiv 0$ due to (1.8). Moreover, if e.g. $i \in\{1, \ldots, N\}$ is such that $p_{i}>q_{i}$, then (1.9) enables us to pick numbers $\theta_{j}>1, j \in\{1, \ldots, N\} \backslash\{i\}$, 
such that $p_{j} \theta_{j}<m_{j}+1$ for all $j \in\{1, \ldots, N\} \backslash\{i\}$ and $\sum_{j \neq i} \frac{1}{\theta_{j}}<1$. An application of Young's inequality thus shows that for any such $i$,

$$
\begin{aligned}
& f_{i}\left(s_{1}, \ldots, s_{N}\right) \geq-\left(p_{i}-q_{i}\right) k_{1} s_{i}^{p_{i}} \cdot \prod_{j \neq i} s_{j}^{p_{j}} \\
& \quad \geq-\left(p_{i}-q_{i}\right) k_{1} s_{i}^{p_{i}} \cdot\left(\sum_{j \neq i} s_{j}^{p_{j} \theta_{j}}+1\right) \quad \text { for all }\left(s_{1}, \ldots, s_{N}\right) \in[0, \infty)^{N},
\end{aligned}
$$

and complementing this by a similar reasoning for all $i \in\{1, \ldots, N\}$ for which $p_{i}<q_{i}$, we readily obtain that (1.5) holds and Theorem 1.1 becomes applicable so as to yield the claim.

Proposition 1.2 corresponds to [15, Remark 2.10], where the existence of weak solutions is proved. The main difference is that there the summation in (1.9) and (1.10) extends over all $j \in\{1, \ldots N\}$.

For linear diffusion, weak solutions of (1.7) have been found in [23] if the reaction functions grow at most quadratically or if the diffusion coefficients are sufficiently close to each other. The same article also deals with their exponential convergence.

Application \#2. A second application of our general theory is concerned with the variant of (1.7) given by

$$
\begin{cases}\partial_{t} u_{1}=d_{1} \Delta u_{1}^{m_{1}}+u_{1}^{\beta_{1}} g_{2}\left(u_{2}\right)-g_{1}\left(u_{1}\right) u_{2}^{\beta_{2}}, & x \in \Omega, t>0, \\ \partial_{t} u_{2}=d_{2} \Delta u_{2}^{m_{2}}-u_{1}^{\beta_{1}} g_{2}\left(u_{2}\right)+\lambda g_{1}\left(u_{1}\right) u_{2}^{\beta_{2}}, & x \in \Omega, t>0, \\ \partial_{\nu} u_{1}^{m_{1}}=\partial_{\nu} u_{2}^{m_{2}}=0, & x \in \partial \Omega, t>0, \\ u_{1}(x, 0)=u_{01}(x), \quad u_{2}(x, 0)=u_{02}(x), & x \in \Omega,\end{cases}
$$

and underlines the mildness of the assumptions in Theorem 1.1 by admitting widely arbitrary growth of the main ingredients $g_{1}$ and $g_{2}$ appearing herein:

Proposition 1.3. Let $d_{1}>0, d_{2}>0, m_{1}>0, m_{2}>0$ and $\lambda \in[0,1]$, let $\beta_{1} \in$ $\left[1, m_{1}+1\right)$ and $\beta_{2} \in\left[1, m_{2}+1\right)$, and let $g_{1} \in C^{1}([0, \infty))$ and $g_{2} \in C^{1}([0, \infty))$ be such that $g_{1}(0)=g_{2}(0)=0$ and that $g_{1}$ and $g_{2}$ are positive on $(0, \infty)$. Then for any pair $\left(u_{01}, u_{02}\right)$ satisfying (1.6), there exists a generalized solution of $(1.11)$ in the spirit of Definition 2.2.

Proof of Proposition 1.3. Taking any nonnegative $\phi_{i} \in C^{1}([0, \infty))$ such that $\phi_{i}^{\prime}>0$ and $\phi_{i} \geq g_{i}$ on $(0, \infty)$ for $i \in\{1,2\}$, one can readily verify that for

$$
\begin{aligned}
f_{1}\left(s_{1}, s_{2}\right):= & s_{1}^{\beta_{1}} g_{2}\left(s_{2}\right)-g_{1}\left(s_{1}\right) s_{2}^{\beta_{2}} \text { and } f_{2}\left(s_{1}, s_{2}\right):=-s_{1}^{\beta_{1}} g_{2}\left(s_{2}\right)+\lambda g_{1}\left(s_{1}\right) s_{2}^{\beta_{2}}, \\
& \left(s_{1}, s_{2}\right) \in[0, \infty)^{2},
\end{aligned}
$$

we have

$$
f_{1}\left(s_{1}, s_{2}\right)+f_{2}\left(s_{1}, s_{2}\right)=-(1-\lambda) g_{1}\left(s_{1}\right) s_{2}^{\beta_{2}} \leq 0 \quad \text { for all }\left(s_{1}, s_{2}\right) \in[0, \infty)^{2}
$$


as well as

$$
f_{1}\left(s_{1}, s_{2}\right) \geq-g_{1}\left(s_{1}\right) s_{2}^{\beta_{2}} \geq-\phi_{1}\left(s_{1}\right) s_{2}^{\beta_{2}} \quad \text { for all }\left(s_{1}, s_{2}\right) \in[0, \infty)^{2}
$$

and, similarly,

$$
f_{2}\left(s_{1}, s_{2}\right) \geq-\phi_{2}\left(s_{2}\right) s_{1}^{\beta_{1}} \quad \text { for all }\left(s_{1}, s_{2}\right) \in[0, \infty)^{2} .
$$

The assumptions $\beta_{i}<m_{i}+1, i \in\{1,2\}$, therefore warrant applicability of Theorem 1.1 with the intended result.

Let us remark that since in Proposition 1.3 not only $f_{1}+f_{2} \leq 0$, but also $\lambda f_{1}\left(s_{1}, s_{2}\right)+$ $f_{2}\left(s_{1}, s_{2}\right)=(\lambda-1) s_{1}^{\beta_{1}} g_{2}\left(s_{2}\right) \leq 0$ for $\left(s_{1}, s_{2}\right) \in[0, \infty)^{2},[15$, Cor. 2.11] could be applied to the variant of (1.11) involving homogeneous Dirichlet boundary conditions (cf. [15, Remark 2.12]) so as to yield weak solutions for any $L^{1}$-initial data; said corollary, however, requires that $m_{1}, m_{2}<2$.

Application \#3. We shall next briefly address

$$
\begin{cases}\partial_{t} u_{1}=d_{1} \Delta u_{1}^{m_{1}}+k_{2} u_{1}^{q_{1}} u_{2}^{q_{2}}-k_{1} u_{1}^{p_{1}} u_{2}^{p_{2}}, & x \in \Omega, t>0, \\ \partial_{t} u_{2}=d_{2} \Delta u_{2}^{m_{2}}-k_{2} u_{1}^{q_{1}} u_{2}^{q_{2}}+k_{1} u_{1}^{p_{1}} u_{2}^{p_{2}}, & x \in \Omega, t>0, \\ \partial_{\nu} u_{1}^{m_{1}}=\partial_{\nu} u_{2}^{m_{2}}=0, & x \in \partial \Omega, t>0 \\ u_{1}(x, 0)=u_{01}(x), \quad u_{2}(x, 0)=u_{02}(x), & x \in \Omega\end{cases}
$$

for which without imposing any smallness condition on $q_{2}$ nor $p_{1}$ we obtain the following.

Corollary 1.4. Let $k_{1}, k_{2}, d_{1}, d_{2}, m_{1}$ and $m_{2}$ be positive, and let $p_{1} \geq 1, p_{2} \geq 1, q_{1} \geq$ 1 and $q_{2} \geq 1$ be such that

$$
q_{1}<m_{1}+1 \quad \text { and } \quad p_{2}<m_{2}+1 \text {. }
$$

Then for all $\left(u_{01}, u_{02}\right)$ fulfilling (1.6), the problem (1.12) possesses a generalized solution in the sense of Definition 2.2.

Proof of Corollary 1.4. With $g_{1}(s)=k_{1} s^{p_{1}}, g_{2}(s)=k_{2} s^{q_{2}}, \beta_{1}=q_{1}, \beta_{2}=p_{2}$, this immediately results from Proposition 1.3.

Application \#4. As final example, let us consider the generalized Lotka-Volterra system

$$
\begin{cases}\partial_{t} u_{i}=d_{i} \Delta u_{i}^{m_{i}}+\gamma_{i} u_{i}+\sum_{j=1}^{N} a_{i j} u_{j}^{\beta_{i j}} u_{i}^{\beta_{j i}}, & x \in \Omega, t>0, i \in\{1, \ldots, N\}, \\ \partial_{\nu} u_{i}^{m_{i}}=0, & x \in \partial \Omega, t>0, i \in\{1, \ldots, N\}, \\ u_{i}(x, 0)=u_{0 i}(x), & x \in \Omega, i \in\{1, \ldots, N\},\end{cases}
$$

which does not obey the typical entropy condition (that is required for the renormalized solutions in [10] and for classical solvability e.g. in [26]). In [9], global classical solutions are shown to exist for the classical Lotka-Volterra system $\left(\beta_{i j}=1\right.$ for all 
$i, j)$ with linear diffusion. If $m_{i}=1$ for all $i$ and $\beta_{i j}=\beta$ for all $i, j$, then the result of [9] covers $2 \beta<2+\varepsilon$ (for sufficiently small $\varepsilon \in(0,1)$, see [9, Step 1, (7)]).

Within the generalized solvability framework considered here, the following consequence of Theorem 1.1 shows that here actually the entire range $\beta<2$ can be exhausted.

Proposition 1.5. Let $N \geq 2, d_{i}>0, m_{i}>0$ and $\gamma_{i} \in \mathbb{R}$ for $i \in\{1, \ldots, N\}$, and suppose that for $i, j \in\{1, \ldots, N\}$ the numbers $a_{i j} \in \mathbb{R}$ and $\beta_{i j}>0$ are such that $a_{i j}+a_{j i} \leq 0$, and that

$$
\text { if } i \neq j \text { and } a_{i j}<0, \quad \text { then } \beta_{i j}<m_{i}+1 \text {. }
$$

Then for all initial data $u_{01}, \ldots, u_{0 n}$ as in (1.6), (1.13) has a generalized solution in the sense of Definition 2.2.

Proof of Proposition 1.5. With $f_{i}(s)=\gamma_{i} s_{i}+\sum_{j=1}^{N} a_{i j} s_{j}^{\beta_{i j}} s_{i}^{\beta_{j i}}, i \in\{1, \ldots, N\},(1.2)$ is clearly satisfied. As

$$
\begin{aligned}
\sum_{i=1}^{N} f_{i}(s)= & \sum_{i=1}^{N} \gamma_{i} s_{i}+\sum_{i=1}^{N} \sum_{j=1}^{N} a_{i j} s_{j}^{\beta_{i j}} s_{i}^{\beta_{j i}} \leq \sum_{i=1}^{N} \gamma_{i} s_{i}+\sum_{i<j}\left(a_{i j} s_{j}^{\beta_{i j}} s_{i}^{\beta_{j i}}\right. \\
& \left.+a_{j i} s_{i}^{\beta_{j i}} s_{j}^{\beta_{i j}}\right) \leq \max _{i} \gamma_{i} \sum_{i=1}^{N} s_{i}
\end{aligned}
$$

due to the fact that $a_{i i} \leq 0$ for all $i \in\{1, \ldots, N\}$, we see that also (1.3) holds. Finally,

$$
\begin{aligned}
f_{i}(s) & \geq \gamma_{i} s_{i}+\sum_{\substack{j \in\{1, \ldots, N\} \\
a_{i j}<0}} a_{i j} s_{j}^{\beta_{i j}} s_{i}^{\beta_{j i}} \\
& \geq-\left(\left|\gamma_{i}\right|+\left|a_{i i}\right| s_{i}^{\beta_{i i}}+\sum_{\substack{j \in\{1, \ldots, N\} \backslash\{i\} \\
a_{i j}<0}}\left|a_{i j}\right| s_{j}^{\beta_{i j}}\right)\left(s_{i}+\sum_{j=1}^{N} s_{i}^{\beta_{j i}}\right) \\
& \geq-\phi_{i}\left(s_{i}\right)\left(1+\sum_{\substack{j \in\{1, \ldots, N\} \backslash\{i\} \\
a_{i j}<0}} s_{j}^{\beta_{i j}}\right)
\end{aligned}
$$

for all $s=\left(s_{1}, \ldots, s_{N}\right) \in[0, \infty)^{N}$ if we set $\phi_{i}\left(s_{i}\right)=\max \left\{\left|\gamma_{i}\right|,\left|a_{i j}\right| \mid j \in\{1, \ldots N\}\right\}$. $\left(1+s_{i}^{\left.\beta_{i i}\right)}\left(s_{i}+\sum_{j=1}^{N} s_{i}^{\beta_{j i}}\right)\right.$ for any such $s$, so that, according to (1.14), (1.5) is fulfilled and Theorem 1.1 is applicable.

\section{Solution concept}

The first step toward the design of our solution concept is concerned with an appropriate supersolution feature required in each of the equations making up (1.4): 
Definition 2.1. Suppose that for $i \in\{1, \ldots, N\}, u_{0 i}: \Omega \rightarrow \mathbb{R}$ and $u_{i}: \Omega \times(0, \infty) \rightarrow$ $\mathbb{R}$ are measurable and nonnegative. Then $\left(u_{1}, \ldots, u_{N}\right)$ will be called a renormalized supersolution of (1.4) if for every $\rho \in C^{\infty}([0, \infty))$ fulfilling $\rho^{،} \in C_{0}^{\infty}([0, \infty))$, $\rho^{\prime} \leq 0$ and $\rho^{\prime \prime} \geq 0$, with

$$
\begin{aligned}
P_{i}^{(1)}(s): & \int_{0}^{s} \sigma^{\frac{m_{i}-1}{2}} \sqrt{\rho^{\prime \prime}(\sigma)} d \sigma \text { and } P_{i}^{(2)}(s):=\int_{0}^{s} \sigma^{m_{i}-1} \rho^{\prime}(\sigma) d \sigma, \\
& s \geq 0, i \in\{1, \ldots, N\},
\end{aligned}
$$

we have

$$
\begin{aligned}
& \rho^{\prime}\left(u_{i}\right) f_{i}\left(u_{1}, \ldots, u_{N}\right) \in L_{l o c}^{1}(\bar{\Omega} \times[0, \infty)) \text { and } \nabla P_{i}^{(1)}\left(u_{i}\right) \in L_{l o c}^{2}\left(\bar{\Omega} \times[0, \infty) ; \mathbb{R}^{n}\right) \\
& \quad \text { for all } i \in\{1, \ldots, N\},
\end{aligned}
$$

and if moreover

$$
\begin{aligned}
-\int_{0}^{\infty} \int_{\Omega} \rho\left(u_{i}\right) \varphi_{t}-\int_{\Omega} \rho\left(u_{0 i}\right) \varphi(\cdot, 0) \leq & -d_{i} m_{i} \int_{0}^{\infty} \int_{\Omega} \varphi\left|\nabla P_{i}^{(1)}\left(u_{i}\right)\right|^{2} \\
& +d_{1} m_{i} \int_{0}^{\infty} \int_{\Omega} P_{i}^{(2)}\left(u_{i}\right) \Delta \varphi \\
& +\int_{0}^{\infty} \int_{\Omega} \rho^{\prime}\left(u_{i}\right) f_{i}\left(u_{1}, \ldots, u_{N}\right) \varphi
\end{aligned}
$$

for all $i \in\{1, \ldots, N\}$ and each nonnegative $\varphi \in C_{0}^{\infty}(\bar{\Omega} \times[0, \infty))$ fulfilling $\partial_{\nu} \varphi=0$ on $\partial \Omega \times(0, \infty)$.

Remark. (i) In the above situation, both integrals on the left of (2.3) as well as the second integral on the right-hand side therein exist due to the readily verified fact that $\rho$ and $P_{i}^{(2)}, i \in\{1, \ldots, N\}$, are bounded on $[0, \infty)$.

(ii) The supersolution property in [15, Prop. 3.6] is obtained upon the choice of $\rho(x)=x$ (inadmissible in Definition 2.1), integration by parts in the integral involving $P_{i}^{(2)}$ (and addition of a corresponding integrability requirement) and, finally, exchange of $\leq$ by $\geq$ (resulting from the change of sign of $\rho^{\prime}$ ).

(iii) The reason for dealing with supersolutions, that is, an inequality instead of an equality in (2.3), lies in the treatment of the integral containing $\left|\nabla P_{i}^{(1)}\left(u_{i}\right)\right|^{2}$, which will be estimated by means of lower semicontinuity, cf. (3.28).

(iv) The definition of $P_{i}^{(1)}$, crucial in making use of the comparatively weak limit information for gradients, c.f. iii), contains $\sqrt{\rho^{\prime \prime}}$, thereby causing the requirement $\rho^{\prime \prime} \geq 0$ - and, consequently, due to compact support of $\rho^{\prime}$ also $\rho^{\prime} \leq 0-$, whereas comparable definitions in other systems only ask for the renormalization to be smooth with compactly supported derivative.

As discussed in several previous related approaches toward generalized solvability on the basis of supersolution features of the above flavor [28,29], supplementing 
Definition 2.1 by a mere requirement on mass control is already sufficient to create a notion of solvability which within classes of suitably smooth functions indeed reduces to classical ones (see, e.g, [17] and [27] for detailed reasonings in this regard):

Definition 2.2. By a generalized solution of (1.4) we mean a vector $\left(u_{1}, \ldots, u_{N}\right)$ of nonnegative measurable functions on $\Omega \times(0, \infty)$ such that $\left(u_{1}, \ldots, u_{N}\right)$ is a renormalized supersolution of (1.4) in the sense of Definition 2.1, that with some $a \in(0, \infty)^{N}$

$$
u_{1}, \ldots, u_{N} \text { and } \sum_{i=1}^{N} a_{i} f_{i}\left(u_{1}, \ldots, u_{N}\right) \text { belong to } L_{l o c}^{1}(\bar{\Omega} \times[0, \infty)),
$$

and that

$$
\int_{\Omega}\left(\sum_{i=1}^{N} a_{i} u_{i}(\cdot, t)\right) \leq \int_{\Omega}\left(\sum_{i=1}^{N} a_{i} u_{0 i}\right)+\int_{0}^{t} \int_{\Omega}\left(\sum_{i=1}^{N} a_{i} f_{i}\left(u_{1}, \ldots, u_{N}\right)\right)
$$

for a.e. $t>0$.

\section{Approximate systems}

In order to construct such solutions through an essentially standard type of approximation, for $\varepsilon \in(0,1)$ we consider

$$
\begin{cases}\partial_{t} u_{i \varepsilon}=d_{i} \Delta\left(u_{i \varepsilon}+\varepsilon\right)^{m_{i}}+\frac{f_{i}\left(u_{1 \varepsilon}, \ldots, u_{N \varepsilon}\right)}{1+\varepsilon \sum_{j=1}^{N}\left|f_{j}\left(u_{1 \varepsilon}, \ldots, u_{N \varepsilon}\right)\right|}, & x \in \Omega, t>0, i \in\{1, \ldots, N\}, \\ \partial_{\nu} u_{i \varepsilon}=0, & x \in \partial \Omega, t>0, i \in\{1, \ldots, N\}, \\ u_{i \varepsilon}(x, 0)=u_{0 i \varepsilon}(x), & x \in \Omega, i \in\{1, \ldots, N\},\end{cases}
$$

where

$$
\left\{\begin{array}{l}
\left(u_{0 i \varepsilon}\right)_{\varepsilon \in(0,1)} \subset C^{1}(\bar{\Omega}) \quad \text { is such that } u_{0 i \varepsilon} \geq 0 \text { for all } i \in\{1, \ldots, N\}, \text { that } \\
u_{0 i \varepsilon} \rightarrow u_{0 i} \text { in } L^{1}(\Omega) \text { and a.e. in } \Omega \text { as } \varepsilon \searrow 0 \text { for all } i \in\{1, \ldots, N\}, \text { and that } \\
\sup _{\varepsilon \in(0,1)}\left\|u_{0 i \varepsilon}\right\|_{L^{r}(\Omega)}<\infty
\end{array}\right.
$$

with $r \geq 1$ taken from (1.6).

Due to boundedness of the reaction term therein and nondegeneracy of the diffusion, by [1, Theorems 14.4 and 14.6] (for local existence) and [16, Theorems V.7.3 and V.7.2] (for a priori bounds ensuring extensibility to globally defined solutions), for each fixed $\varepsilon \in(0,1)$ the problem $(3.1)$ indeed admits a global classical solution

$$
u_{i \varepsilon} \in C^{0}(\bar{\Omega} \times[0, \infty)) \cap C^{2,1}(\bar{\Omega} \times(0, \infty)) \quad \text { for all } i \in\{1, \ldots, N\},
$$

which, moreover, is nonnegative.

General assumption. Throughout the sequel, we shall suppose that the assumptions of Theorem 1.1 and (3.2) are satisfied, and given $\varepsilon \in(0,1)$ we let $\left(u_{1 \varepsilon}, \ldots, u_{N \varepsilon}\right)$ denote the global classical solution of (3.1). 
The following basic observation concerning $L^{1}$-boundedness of these solutions is a fairly immediate consequence of (1.3).

Lemma 3.1. For all $T>0$ there exists $C(T)>0$ such that for all $i \in\{1, \ldots, N\}$ and any $\varepsilon \in(0,1)$ we have

$$
\left\|u_{i \varepsilon}(\cdot, t)\right\|_{L^{1}(\Omega)} \leq C(T) \quad \text { for all } t \in(0, T) .
$$

Proof. By integrating in (3.1), we see that since $\partial_{\nu} u_{i \varepsilon}=0$ on $\partial \Omega \times(0, \infty)$, due to (1.3) we have

$$
\begin{aligned}
\frac{d}{d t} \sum_{i=1}^{N} a_{i} \int_{\Omega} u_{i \varepsilon} & =\int_{\Omega} \frac{1}{1+\varepsilon \sum_{j=1}^{N}\left|f_{j}\left(u_{1 \varepsilon}, \ldots, u_{N \varepsilon}\right)\right|} \cdot \sum_{i=1}^{N} a_{i} f_{i}\left(u_{1 \varepsilon}, \ldots, u_{N \varepsilon}\right) \\
& \leq \int_{\Omega} \frac{1}{1+\varepsilon \sum_{j=1}^{N}\left|f_{j}\left(u_{1 \varepsilon}, \ldots, u_{N \varepsilon}\right)\right|} \cdot K\left\{\sum_{i=1}^{N} a_{i} u_{i \varepsilon}+1\right\} \\
& \leq K \sum_{j=1}^{N} a_{i} \int_{\Omega} u_{i \varepsilon}+K|\Omega| \quad \text { for all } t>0 \text { and } \varepsilon \in(0,1),
\end{aligned}
$$

because $u_{i \varepsilon} \geq 0$ for all $i \in\{1, \ldots, N\}$. By an ODE comparison, this shows that

$$
\sum_{i=1}^{N} a_{i} \int_{\Omega} u_{i \varepsilon} \leq\left\{\sum_{i=1}^{N} a_{i} \int_{\Omega} u_{0 i \varepsilon}+|\Omega|\right\} \cdot e^{K t} \quad \text { for all } t>0 \text { and } \varepsilon \in(0,1)
$$

and hence, again by nonnegativity of $u_{i \varepsilon}$ for $i \in\{1, \ldots, N\}$, establishes (3.3) due to (3.2).

The following estimate rests on a duality-based reasoning inspired by a corresponding argument from [15]. An important difference is given by the change of boundary conditions: Where [15] dealt with Dirichlet boundary data and thus could use the solution of Poisson's equation as test function, the non-invertibility of the Laplacian with homogeneous Neumann boundary data leads us to employ the solution of a Helmholtz equation instead. An alternative approach of working with the Neumann Laplacian after subtracting the mean value has been followed in [14], but it seems unclear how far strategies of this type can be applied so as to successfully cover the present setting.

Lemma 3.2. For all $T>0$ there exists $C(T)>0$ such that for all $i \in\{1, \ldots, N\}$,

$$
\int_{0}^{T} \int_{\Omega} u_{i \varepsilon}^{m_{i}+1} \leq C(T) \quad \text { for all } \varepsilon \in(0,1) .
$$

Proof. Following [15, Proof of Theorem 2.7], we first observe that according to (3.1) and (1.3), writing

$$
\begin{aligned}
& w_{\varepsilon}(\cdot, t):=e^{-K t} \cdot \sum_{i=1}^{N} a_{i} u_{i \varepsilon}(\cdot, t) \\
& \text { and } \quad z_{\varepsilon}(\cdot, t):=\int_{0}^{t} e^{-K s} \cdot\left\{\sum_{i=1}^{N} a_{i} d_{i}\left(u_{i \varepsilon}+\varepsilon\right)^{m_{i}}\right\}
\end{aligned}
$$


for $\varepsilon \in(0,1)$ and $t \geq 0$, we have

$$
\begin{aligned}
\partial_{t} w_{\varepsilon}= & e^{-K t} \Delta\left\{\sum_{i=1}^{N} a_{i} d_{i}\left(u_{i \varepsilon}+\varepsilon\right)^{m_{i}}\right\}+e^{-K t} \sum_{i=1}^{N} \frac{a_{i} f_{i}\left(u_{1 \varepsilon}, \ldots, u_{N \varepsilon}\right)}{1+\varepsilon \sum_{j=1}^{N}\left|f_{j}\left(u_{1 \varepsilon}, \ldots, u_{N \varepsilon}\right)\right|} \\
& -K e^{-K t} \sum_{i=1}^{N} a_{i} u_{i \varepsilon} \\
\leq & e^{-K t} \Delta\left\{\sum_{i=1}^{N} a_{i} d_{i}\left(u_{i \varepsilon}+\varepsilon\right)^{m_{i}}\right\}+K e^{-K t} \quad \text { in } \Omega \times(0, \infty) \quad \text { for all } \varepsilon \in(0,1)
\end{aligned}
$$

and hence

$$
w_{\varepsilon}(\cdot, t) \leq w_{\varepsilon}(\cdot, 0)+\Delta z_{\varepsilon}(\cdot, t)+1 \text { in } \Omega \text { for all } t>0 \text { and } \varepsilon \in(0,1),
$$

because $\int_{0}^{t} K e^{-K s} d s=1-e^{-K s} \leq 1$ for all $t>0$. Upon multiplication by $\partial_{t} z_{\varepsilon} \geq 0$ and integration over $\Omega \times(0, T)$ for $T>0$, as in [15] we obtain that since $z_{\varepsilon}(\cdot, 0) \equiv 0$,

$$
\begin{aligned}
\int_{0}^{T} \int_{\Omega} w_{\varepsilon} \partial_{t} z_{\varepsilon} & \leq \int_{\Omega} w_{\varepsilon}(\cdot, 0) z_{\varepsilon}(\cdot, T)-\frac{1}{2} \int_{\Omega}\left|\nabla z_{\varepsilon}(\cdot, T)\right|^{2}+\int_{\Omega} z_{\varepsilon}(\cdot, T) \\
& \leq \int_{\Omega}\left(w_{\varepsilon}(\cdot, 0)+1\right) z_{\varepsilon}(\cdot, T) \quad \text { for all } \varepsilon \in(0,1)
\end{aligned}
$$

where the right-hand side is now estimated in a way slightly deviating from that in [15] due to the different boundary conditions considered here. In fact, by nonnegativity of $w_{\varepsilon}$ the inequality in (3.5) implies that for each fixed $T>0$ and arbitrary $\varepsilon \in(0,1)$,

$$
\left\{\begin{array}{l}
-\Delta z_{\varepsilon}(\cdot, T)+z_{\varepsilon}(\cdot, T) \leq w_{\varepsilon}(\cdot, 0)+1+z_{\varepsilon}(\cdot, T) \text { in } \Omega \quad \text { and } \\
\partial_{\nu} z_{\varepsilon}(\cdot, T)=0 \text { on } \partial \Omega,
\end{array}\right.
$$

so that since the Helmholtz operator $-\Delta+1$ admits a comparison principle under homogeneous Neumann boundary conditions, we obtain that

$$
z_{\varepsilon}(\cdot, T) \leq \overline{z_{\varepsilon}} \quad \text { in } \Omega,
$$

where $\overline{z_{\varepsilon}}$ denotes the solution of

$$
\left\{\begin{array}{l}
-\Delta \overline{z_{\varepsilon}}+\overline{z_{\varepsilon}}=w_{\varepsilon}(\cdot, 0)+1+z_{\varepsilon}(\cdot T) \text { in } \Omega, \\
\partial_{\nu} \overline{z_{\varepsilon}}=0 \text { on } \partial \Omega .
\end{array}\right.
$$

Now without loss of generality assuming that the number $r \geq 1$ in (1.6) satisfies $r<2$, we take $r^{\prime} \in(2, \infty]$ such that $\frac{1}{r}+\frac{1}{r^{\prime}}=1$, and employ a Sobolev embedding theorem and elliptic regularity theory [11] to find $c_{1}>0$ and $c_{2}>0$ such that for all $\varepsilon \in(0,1)$,

$$
\begin{aligned}
\left\|\overline{z_{\varepsilon}}\right\|_{L^{r^{\prime}}(\Omega)} & \leq c_{1}\left\|\overline{z_{\varepsilon}}\right\|_{W^{2, r}(\Omega)} \leq c_{2}\left\|w_{\varepsilon}(\cdot, 0)+1+z_{\varepsilon}(\cdot, T)\right\|_{L^{r}(\Omega)} \\
& \leq c_{2}\left\|w_{\varepsilon}(\cdot, 0)+1\right\|_{L^{r}(\Omega)}+c_{2}\left\|z_{\varepsilon}(\cdot, T)\right\|_{L^{r}(\Omega)}
\end{aligned}
$$


because the restrictions on $r$ in (1.6) warrant that $2-\frac{n}{r} \geq-\frac{n}{r^{\prime}}$ if $n \geq 3$, and that $2-\frac{n}{r}>0$ if $n \leq 2$. Here we note that according to Lemma 3.1 we know that if we let $\theta \in(0,1)$ small enough such that $\theta m_{i} \leq 1$ for all $i \in\{1, \ldots, N\}$, then we can find $c_{3}(T)>0$ fulfilling

$$
\begin{aligned}
\int_{\Omega} z_{\varepsilon}^{\theta}(\cdot, T) & \leq T^{\theta} \sup _{t \in(0, T)} \int_{\Omega}\left\{\sum_{i=1}^{N} a_{i} d_{i}\left(u_{i \varepsilon}(\cdot, t)+\varepsilon\right)^{m_{i}}\right\}^{\theta} \\
& \leq\left\{N T \max _{i \in\{1, \ldots, N\}} a_{i} d_{i}\right\}^{\theta} \max _{i \in\{1, \ldots, N\}} \sup _{t \in(0, T)} \int_{\Omega}\left(u_{i \varepsilon}(\cdot, t)+\varepsilon\right)^{\theta m_{i}} \\
& \leq\left\{N T \max _{i \in\{1, \ldots, N\}} a_{i} d_{i}\right\}^{\theta} \max _{i \in\{1, \ldots, N\}} \sup _{t \in(0, T)} \int_{\Omega}\left(u_{i \varepsilon}(\cdot, t)+1\right) \\
& \leq c_{3}(T) \quad \text { for all } \varepsilon \in(0,1),
\end{aligned}
$$

whence using that then $\theta<1<r<2<r^{\prime}$ we may invoke Hölder's and Young's inequality to see that with $\lambda=\left(\frac{1}{\theta}-\frac{1}{r}\right) /\left(\frac{1}{\theta}-\frac{1}{r^{\prime}}\right) \in(0,1)$ and some $c_{4}>0$ we have

$$
\begin{aligned}
c_{2}\left\|z_{\varepsilon}(\cdot, T)\right\|_{L^{r}(\Omega)} & \leq c_{2}\left\|z_{\varepsilon}(\cdot, T)\right\|_{L^{r^{\prime}}(\Omega)}^{\lambda}\left\|z_{\varepsilon}(\cdot, T)\right\|_{L^{\theta}(\Omega)}^{1-\lambda} \\
& \leq \frac{1}{2}\left\|z_{\varepsilon}(\cdot, T)\right\|_{L^{r^{\prime}(\Omega)}}+c_{4}\left\|z_{\varepsilon}(\cdot, T)\right\|_{L^{\theta}(\Omega)} \\
& \leq \frac{1}{2}\left\|z_{\varepsilon}(\cdot, T)\right\|_{L^{r^{\prime}(\Omega)}}+c_{3}^{\frac{1}{\theta}}(T) c_{4} \quad \text { for all } \varepsilon \in(0,1) .
\end{aligned}
$$

In view of (3.7) and the nonnegativity of $z_{\varepsilon}$, (3.8) thus implies that

$$
\begin{aligned}
\left\|z_{\varepsilon}(\cdot, T)\right\|_{L^{r^{\prime}}(\Omega)} \leq & \left\|\overline{z_{\varepsilon}}\right\|_{L^{r^{\prime}}(\Omega)} \leq c_{2}\left\|w_{\varepsilon}(\cdot, 0)+1\right\|_{L^{r}(\Omega)} \\
& +\frac{1}{2}\left\|z_{\varepsilon}(\cdot, T)\right\|_{L^{r^{\prime}}(\Omega)}+c_{3}^{\frac{1}{\theta}}(T) c_{4} \quad \text { for all } \varepsilon \in(0,1),
\end{aligned}
$$

so that in (3.6) we can use the Hölder inequality to estimate

$$
\begin{aligned}
\int_{\Omega}\left(w_{\varepsilon}(\cdot, 0)+1\right) z_{\varepsilon}(\cdot, T) & \leq\left\|w_{\varepsilon}(\cdot, 0)+1\right\|_{L^{r}(\Omega)}\left\|z_{\varepsilon}(\cdot, T)\right\|_{L^{r^{\prime}}(\Omega)} \\
& \leq\left\|w_{\varepsilon}(\cdot, 0)+1\right\|_{L^{r}(\Omega)} \cdot\left\{2 c_{2}\left\|w_{\varepsilon}(\cdot, 0)+1\right\|_{L^{r}(\Omega)}+2 c_{3}^{\frac{1}{\theta}}(T) c_{4}\right\}
\end{aligned}
$$

for all $\varepsilon \in(0,1)$. As $\sup _{\varepsilon \in(0,1)}\left\|w_{\varepsilon}(\cdot, 0)\right\|_{L^{r}(\Omega)}$ is finite according to the hypothesis (3.2), (3.6) thereby entails the existence of $c_{5}(T)>0$ such that

$$
\int_{0}^{T} \int_{\Omega} e^{-2 K t} \cdot\left\{\sum_{i=1}^{N} d_{i}\left(u_{i \varepsilon}+\varepsilon\right)^{m_{i}}\right\} \cdot\left\{\sum_{i=1}^{N} u_{i \varepsilon}\right\} \leq c_{5}(T) \quad \text { for all } \varepsilon \in(0,1),
$$

from which (3.4) readily follows.

We next rely on (1.5) in deriving the following estimates for gradients and reaction terms. Testing (3.1) by $-\frac{1}{\phi_{i}\left(u_{i \varepsilon}\right)}$, namely, enables us to successfully combine (1.5) with (3.4). In order to obtain a bound for, e.g., $\left|f_{i}\left(u_{1 \varepsilon}, \ldots, u_{N \varepsilon}\right)\right|$ instead of $\left|-\frac{1}{\phi_{i}\left(u_{i \varepsilon}\right)} f_{i}\left(u_{1 \varepsilon}, \ldots, u_{N \varepsilon}\right)\right|$, we here restrict our attention to sets of the form $\left\{u_{i \varepsilon} \leq M\right\}$, where $\left|-\frac{1}{\phi_{i}\left(u_{i \varepsilon}\right)}\right|$ can be estimated from below by a positive constant. 
Lemma 3.3. Let $M>0$ and $T>0$. Then one can find $C(M, T)>0$ such that

$$
\begin{array}{r}
\int_{0}^{T} \int_{\Omega} \chi\left\{u_{i \varepsilon} \leq M\right\} \\
\left(u_{i \varepsilon}+\varepsilon\right)^{m_{i}-1}\left|\nabla u_{i \varepsilon}\right|^{2} \leq C(M, T) \\
\text { for all } i \in \mathbb{N} \text { and } \varepsilon \in(0,1)
\end{array}
$$

and

$$
\begin{array}{r}
\int_{0}^{T} \int_{\Omega} \chi_{\left\{u_{i \varepsilon} \leq M\right\}} \frac{\left|f_{i}\left(u_{1 \varepsilon}, \ldots, u_{N \varepsilon}\right)\right|}{1+\varepsilon \sum_{j=1}^{N}\left|f_{j}\left(u_{1 \varepsilon}, \ldots, u_{N \varepsilon}\right)\right|} \leq C(M, T) \\
\text { for all } i \in \mathbb{N} \text { and } \varepsilon \in(0,1) .
\end{array}
$$

Proof. For fixed $i \in\{1, \ldots, N\}$, we take $\phi_{i} \in C^{1}([0, \infty))$ as in (1.5) and define

$$
\Phi_{i}(s):=-\int_{1}^{s+1} \frac{d \sigma}{\phi_{i}(\sigma)}, \quad s \geq 0 .
$$

Then since $\phi_{i}$ is nondecreasing, we have

$$
0 \geq \Phi_{i}(s) \geq-c_{1 i} s \quad \text { for all } s \geq 0
$$

with $c_{1 i}:=\frac{1}{\phi_{i}(1)}>0$, and moreover $\Phi_{i}^{\prime}(s)=-\frac{1}{\phi_{i}(s+1)}, s \geq 0$, satisfies

$$
0 \leq-\Phi_{i}^{\prime}(s) \leq \frac{1}{\phi_{i}(s)} \quad \text { for all } s \geq 0
$$

Now using (3.1), for $\varepsilon \in(0,1)$ and $t>0$ we compute

$$
\begin{aligned}
\frac{d}{d t} \int_{\Omega} \Phi_{i}\left(u_{i \varepsilon}\right)= & \int_{\Omega} \Phi_{i}^{\prime}\left(u_{i \varepsilon}\right) \cdot\left\{d_{i} m_{i} \nabla \cdot\left(\left(u_{i \varepsilon}+\varepsilon\right)^{m_{i}-1} \nabla u_{i \varepsilon}\right)\right. \\
& \left.+\frac{f_{i}\left(u_{1 \varepsilon}, \ldots, u_{N \varepsilon}\right)}{1+\varepsilon \sum_{j=1}^{N}\left|f_{j}\left(u_{1 \varepsilon}, \ldots, u_{N \varepsilon}\right)\right|}\right\} \\
=- & d_{i} m_{i} \int_{\Omega}\left(u_{i \varepsilon}+\varepsilon\right)^{m_{i}-1} \Phi_{i}^{\prime \prime}\left(u_{i \varepsilon}\right)\left|\nabla u_{i \varepsilon}\right|^{2} \\
& +\int_{\Omega} \Phi_{i}^{\prime}\left(u_{i \varepsilon}\right) \cdot \frac{f_{i}\left(u_{1 \varepsilon}, \ldots, u_{N \varepsilon}\right)}{1+\varepsilon \sum_{j=1}^{N}\left|f_{j}\left(u_{1 \varepsilon}, \ldots, u_{N \varepsilon}\right)\right|},
\end{aligned}
$$

so that splitting $f_{i}=\left|f_{i}\right|-2\left(f_{i}\right)_{-}$, upon further integration we find that

$$
\begin{aligned}
& d_{i} m_{i} \int_{0}^{T} \int_{\Omega}\left(u_{i \varepsilon}+\varepsilon\right)^{m_{i}-1} \Phi_{i}^{\prime \prime}\left(u_{i \varepsilon}\right)\left|\nabla u_{i \varepsilon}\right|^{2} \\
& \quad+\int_{0}^{T} \int_{\Omega}\left|\Phi_{i}^{\prime}\left(u_{i \varepsilon}\right)\right| \cdot \frac{\left|f_{i}\left(u_{1 \varepsilon}, \ldots, u_{N \varepsilon}\right)\right|}{1+\varepsilon \sum_{j=1}^{N}\left|f_{j}\left(u_{1 \varepsilon}, \ldots, u_{N \varepsilon}\right)\right|} \\
& =\int_{\Omega} \Phi_{i}\left(u_{0 i \varepsilon}\right)-\int_{\Omega} \Phi_{i}\left(u_{i \varepsilon}(\cdot, T)\right) \\
& \quad+2 \int_{0}^{T} \int_{\Omega}\left|\Phi_{i}^{\prime}\left(u_{i \varepsilon}\right)\right| \cdot \frac{\left(f_{i}\left(u_{1 \varepsilon}, \ldots, u_{N \varepsilon}\right)\right)_{-}}{1+\varepsilon \sum_{j=1}^{N}\left|f_{j}\left(u_{1 \varepsilon}, \ldots, u_{N \varepsilon}\right)\right|} \quad \text { for all } T>0,
\end{aligned}
$$


because $\Phi_{i}^{\prime}$ is nonpositive. Now on the right-hand side of (3.13) we use (1.5) to see that thanks to (3.12),

$$
\begin{aligned}
& 2 \int_{0}^{T} \int_{\Omega}\left|\Phi_{i}^{\prime}\left(u_{i \varepsilon}\right)\right| \cdot \frac{\left(f_{i}\left(u_{1 \varepsilon}, \ldots, u_{N \varepsilon}\right)\right)_{-}}{1+\varepsilon \sum_{j=1}^{N}\left|f_{j}\left(u_{1 \varepsilon}, \ldots, u_{N \varepsilon}\right)\right|} \leq 2 \int_{0}^{T} \int_{\Omega}\left|\Phi_{i}^{\prime}\left(u_{i \varepsilon}\right)\right| \cdot \phi_{i}\left(u_{i \varepsilon}\right) \cdot\left\{\sum_{j \neq i} u_{j \varepsilon}^{\beta_{j}}+1\right\} \\
& \leq 2 \sum_{j \neq i} \int_{0}^{T} \int_{\Omega} u_{j \varepsilon}^{\beta_{j}}+2|\Omega| T \\
& \text { for all } T>0,
\end{aligned}
$$

whence recalling Lemma 3.2 we obtain $c_{2 i}(T)>0$ such that for all $\varepsilon \in(0,1)$,

$$
2 \int_{0}^{T} \int_{\Omega}\left|\Phi_{i}^{\prime}\left(u_{i \varepsilon}\right)\right| \cdot \frac{\left(f_{i}\left(u_{1 \varepsilon}, \ldots, u_{N \varepsilon}\right)\right)_{-}}{1+\varepsilon \sum_{j=1}^{N}\left|f_{j}\left(u_{1 \varepsilon}, \ldots, u_{N \varepsilon}\right)\right|} \leq c_{2 i}(T) .
$$

As

$$
\int_{\Omega} \Phi_{i}\left(u_{0 i \varepsilon}\right)-\int_{\Omega} \Phi_{i}\left(u_{i \varepsilon}(\cdot, T)\right) \leq c_{1 i} \int_{\Omega} u_{i \varepsilon}(\cdot, T) \quad \text { for all } T>0
$$

by (3.11), in view of Lemma 3.1 we thus conclude from (3.13) that there exists $c_{3 i}(T)>$ 0 with the property that

$$
\begin{gathered}
d_{i} m_{i} \int_{0}^{T} \int_{\Omega}\left(u_{i \varepsilon}+\varepsilon\right)^{m_{i}-1} \Phi_{i}^{\prime \prime}\left(u_{i \varepsilon}\right)\left|\nabla u_{i \varepsilon}\right|^{2}+\int_{0}^{T} \int_{\Omega}\left|\Phi_{i}^{\prime}\left(u_{i \varepsilon}\right)\right| \\
\cdot \frac{\left|f_{i}\left(u_{1 \varepsilon}, \ldots, u_{N \varepsilon}\right)\right|}{1+\varepsilon \sum_{j=1}^{N}\left|f_{j}\left(u_{1 \varepsilon}, \ldots, u_{N \varepsilon}\right)\right|} \leq c_{3 i}(T)
\end{gathered}
$$

for all $\varepsilon \in(0,1)$. Noting that for fixed $M>0$ and any $i \in\{1, \ldots, N\}$ the numbers

$$
\begin{aligned}
c_{4 i} & :=\min _{s \in[0, M]} \Phi_{i}^{\prime \prime}(s)=\min _{s \in[0, M]} \frac{\phi_{i}^{\prime}(s+1)}{\phi_{i}^{2}(s+1)} \quad \text { and } \\
c_{5 i} & :=\min _{s \in[0, M]}\left|\Phi_{i}^{\prime}(s)\right|=\frac{1}{\phi_{i}(M+1)}
\end{aligned}
$$

are both positive according to our hypotheses on $\phi_{i}$ and $\phi_{i}^{\prime}$, from (3.15) we readily infer (3.9) and (3.10) if we let $C(M, T):=\max \left\{\max _{i \in\{1, \ldots, N\}} \frac{c_{3 i}(T)}{d_{i} m_{i} c_{4 i}}, \max _{i \in\{1, \ldots, N\}}\right.$ $\left.\frac{c_{3 i}(T)}{c_{5 i}}\right\}$, for instance.

While the bound in Lemma 3.2 is sufficient for concluding relative compactness of $\left\{u_{i \varepsilon} \mid \varepsilon \in(0,1)\right\}$ in some weak topology, we are additionally interested in possible pointwise convergence of $u_{i \varepsilon_{j}}$ along some sequence $\left(\varepsilon_{j}\right)_{j \in \mathbb{N}} \searrow 0$. We thus strive to derive a suitable strong compactness property in $L^{2}(\Omega \times(0, T))$, at least of a power of $u_{i \varepsilon}$ which has been cut off at large values so as to ensure accessibility to the estimates of Lemma 3.3. 
Lemma 3.4. Given $\zeta \in C_{0}^{\infty}([0, \infty))$, for $i \in\{1, \ldots, N\}$ let $\rho_{i}(s):=s^{\kappa_{i}} \zeta(s), s \geq 0$, where $\kappa_{i}:=\max \left\{\frac{m_{i}+1}{2}, 2\right\}$. Then

$$
\left(\rho_{i}\left(u_{i \varepsilon}\right)\right)_{\varepsilon \in(0,1)} \text { is relatively compact in } L^{2}(\Omega \times(0, T)) \text { for all } T>0 .
$$

Proof. Let us first make sure that for each $T>0$,

$$
\left(\rho_{i}\left(u_{i \varepsilon}\right)\right)_{\varepsilon \in(0,1)} \text { is bounded in } L^{2}\left((0, T) ; W^{1,2}(\Omega)\right) .
$$

To see this, we note that due to the compactness of supp $\rho_{i}$ it is clear that $\left(\rho_{i}\left(u_{i \varepsilon}\right)\right)_{\varepsilon \in(0,1)}$ is bounded in $L^{\infty}(\Omega \times(0, \infty))$. Therefore, (3.17) results upon the observation that if we fix $M>0$ such that $\zeta \equiv 0$ on $(M, \infty)$, then by Young's inequality and (3.9) we see that for all $T>0$ there exists $c_{1}(T)>0$ such that

$$
\begin{aligned}
\int_{0}^{T} \int_{\Omega}\left|\nabla \rho_{i}\left(u_{i \varepsilon}\right)\right|^{2}= & \int_{0}^{T} \int_{\Omega}\left(\rho_{i}^{\prime}\left(u_{i \varepsilon}\right)\right)^{2}\left|\nabla u_{i \varepsilon}\right|^{2} \\
= & \int_{0}^{T} \int_{\Omega}\left(\kappa_{i} u_{i \varepsilon}^{\kappa_{i}-1} \zeta\left(u_{i \varepsilon}\right)+u_{i \varepsilon}^{\kappa_{i}} \zeta^{\prime}\left(u_{i \varepsilon}\right)\right)^{2}\left|\nabla u_{i \varepsilon}\right|^{2} \\
\leq & 2 \kappa_{i}^{2}\|\zeta\|_{L^{\infty}((0, \infty))}^{2} \int_{0}^{T} \int_{\Omega} \chi_{\left\{u_{i \varepsilon} \leq M\right\}} u_{i \varepsilon}^{2 \kappa_{i}-2}\left|\nabla u_{i \varepsilon}\right|^{2} \\
& +2\left\|\zeta^{\prime}\right\|_{L^{\infty}((0, \infty))}^{2} \int_{0}^{T} \int_{\Omega} \chi_{\left\{u_{i \varepsilon} \leq M\right\}} u_{i \varepsilon}^{2 \kappa_{i}}\left|\nabla u_{i \varepsilon}\right|^{2} \\
\leq & 2 \kappa_{i}^{2}\|\zeta\|_{L^{\infty}((0, \infty))}^{2} M^{2 \kappa_{i}-m_{i}-1} \int_{0}^{T} \int_{\Omega} \chi_{\left\{u_{i \varepsilon} \leq M\right\}} u_{i \varepsilon}^{m_{i}-1}\left|\nabla u_{i \varepsilon}\right|^{2} \\
& +2\left\|\zeta^{\prime}\right\|_{L^{\infty}((0, \infty))}^{2} M^{2 \kappa_{i}-m_{i}+1} \int_{0}^{T} \int_{\Omega} \chi_{\left\{u_{i \varepsilon} \leq M\right\}} u_{i \varepsilon}^{m_{i}-1}\left|\nabla u_{i \varepsilon}\right|^{2} \\
\leq & c_{1}(T) \quad \text { for all } \varepsilon \in(0,1),
\end{aligned}
$$

where we have used that $2 \kappa_{i}-m_{i}+1 \geq 2 \kappa_{i}-m_{i}-1 \geq 0$ by hypothesis.

We next fix any integer $k \geq 1$ such that $k>n$, and claim that then for all $T>0$,

$$
\left(\partial_{t} \rho_{i}\left(u_{i \varepsilon}\right)\right)_{\varepsilon \in(0,1)} \text { is bounded in } L^{1}\left((0, T) ;\left(W^{k, 2}(\Omega)\right)^{\star}\right) .
$$

To verify this in quite a straightforward manner, we pick $\psi \in C^{\infty}(\bar{\Omega})$ and use (3.1) to see that for each $t>0$ and any $\varepsilon \in(0,1)$,

$$
\begin{aligned}
& \left|\int_{\Omega} \partial_{t} \rho_{i}\left(u_{i \varepsilon}(\cdot, t)\right) \cdot \psi\right| \\
& =\left|\int_{\Omega} \rho_{i}^{\prime}\left(u_{i \varepsilon}\right) \psi \cdot\left\{d_{i} m_{i} \nabla \cdot\left(\left(u_{i \varepsilon}+\varepsilon\right)^{m_{i}-1} \nabla u_{i \varepsilon}\right)+\frac{f_{i}\left(u_{1 \varepsilon}, \ldots, u_{N \varepsilon}\right)}{1+\varepsilon \sum_{j=1}^{N}\left|f_{j}\left(u_{1 \varepsilon}, \ldots, u_{N \varepsilon}\right)\right|}\right\}\right| \\
& =\left.\left|-d_{i} m_{i} \int_{\Omega}\left(u_{i \varepsilon}+\varepsilon\right)^{m_{i}-1} \rho_{i}^{\prime \prime}\left(u_{i \varepsilon}\right)\right| \nabla u_{i \varepsilon}\right|^{2} \psi-d_{i} m_{i} \int_{\Omega}\left(u_{i \varepsilon}+\varepsilon\right)^{m_{i}-1} \rho_{i}^{\prime}\left(u_{i \varepsilon}\right) \nabla u_{i \varepsilon} \cdot \nabla \psi \\
& \quad+\int_{\Omega} \rho_{i}^{\prime}\left(u_{i \varepsilon}\right) \cdot \frac{f_{i}\left(u_{1 \varepsilon}, \ldots, u_{N \varepsilon}\right)}{1+\varepsilon \sum_{j=1}^{N}\left|f_{j}\left(u_{1 \varepsilon}, \ldots, u_{N \varepsilon}\right)\right|} \cdot \psi \mid
\end{aligned}
$$




$$
\begin{aligned}
& \leq d_{i} m_{i}\left\|\rho_{i}^{\prime \prime}\right\|_{L^{\infty}((0, \infty))} \cdot\left\{\int_{\Omega} \chi_{\left\{u_{i \varepsilon} \leq M\right\}}\left(u_{i \varepsilon}+\varepsilon\right)^{m_{i}-1}\left|\nabla u_{i \varepsilon}\right|^{2}\right\} \cdot\|\psi\|_{L^{\infty}(\Omega)} \\
& \quad+d_{i} m_{i} \cdot\left\{\int_{\Omega} \chi_{\left\{u_{i \varepsilon} \leq M\right\}}\left(u_{i \varepsilon}+\varepsilon\right)^{m_{i}-1}\left|\nabla u_{i \varepsilon}\right|^{2}+|\Omega| \sup _{s \geq 0}(s+\varepsilon)^{m_{i}-1}\left|\rho_{i}^{\prime}(s)\right|^{2}\right\}\|\nabla \psi\|_{L^{\infty}(\Omega)} \\
& +\left\|\rho_{i}^{\prime}\right\|_{L^{\infty}((0, \infty))} \cdot\left\{\int_{\Omega} \chi_{\left\{u_{i \varepsilon} \leq M\right\}} \frac{\left|f_{i}\left(u_{1 \varepsilon}, \ldots, u_{N \varepsilon}\right)\right|}{1+\varepsilon \sum_{j=1}^{N}\left|f_{j}\left(u_{1 \varepsilon}, \ldots, u_{N \varepsilon}\right)\right|}\right\} \cdot\|\psi\|_{L^{\infty}(\Omega)},
\end{aligned}
$$

where finiteness of both $\left\|\rho^{\prime}\right\|_{L^{\infty}((0, \infty))}$ and $\left\|\rho_{i}^{\prime \prime}\right\|_{L^{\infty}((0, \infty))}$ is asserted by our restriction that $\kappa_{i} \geq 2$. Here we observe that in the case $m_{i} \geq 1$ we have

$$
(s+\varepsilon)^{m_{i}-1}\left|\rho_{i}^{\prime}(s)\right|^{2} \leq(M+1)^{m_{i}-1}\left\|\rho_{i}^{\prime}\right\|_{L^{\infty}((0, \infty))}^{2},
$$

whereas if $m_{i}<1$ then

$$
\begin{aligned}
(s+\varepsilon)^{m_{i}-1}\left|\rho_{i}^{\prime}(s)\right|^{2} & \leq s^{m_{i}-1}\left|\rho_{i}^{\prime}(s)\right|^{2} \\
& =s^{m_{i}-1} \cdot\left|\kappa_{i} s^{\kappa_{i}-1} \zeta(s)+s^{\kappa_{i}} \zeta^{\prime}(s)\right|^{2} \\
& \leq 2 \kappa_{i}^{2} s^{2 \kappa_{i}+m_{i}-3} \zeta^{2}(s)+2 s^{2 \kappa_{i}+m_{i}-1}\left|\zeta^{\prime}(s)\right|^{2} \\
& \leq 2 \kappa_{i}^{2} M^{2 \kappa_{i}+m_{i}-3}\|\zeta\|_{L^{\infty}((0, \infty))}^{2}+2 M^{2 \kappa_{i}+m_{i}-1}\left\|\zeta^{\prime}\right\|_{L^{\infty}((0, \infty))}^{2}
\end{aligned}
$$

because again by definition of $\kappa_{i}$, we have $2 \kappa_{i}+m_{i}-1 \geq 2 \kappa_{i}+m_{i}-3 \geq 0$. As furthermore $W^{k, 2}(\Omega) \hookrightarrow W^{1, \infty}(\Omega)$ due to our restriction that $k>n$, from (3.19) we thus infer the existence of $c_{2}>0$ such that for all $t>0$ and any $\varepsilon \in(0,1)$,

$$
\begin{aligned}
\left\|\partial_{t} \rho_{i}\left(u_{i \varepsilon}(\cdot, t)\right)\right\|_{\left(W^{k, 2}(\Omega)\right)^{\star}} \leq & c_{2} \int_{\Omega} \chi_{\left\{u_{i \varepsilon} \leq M\right\}}\left(u_{i \varepsilon}+\varepsilon\right)^{m_{i}-1}\left|\nabla u_{i \varepsilon}\right|^{2}+c_{2} \\
& +c_{2} \int_{\Omega} \chi_{\left\{u_{i \varepsilon} \leq M\right\}} \frac{\left|f_{i}\left(u_{1 \varepsilon}, \ldots, u_{N \varepsilon}\right)\right|}{1+\varepsilon \sum_{j=1}^{N}\left|f_{j}\left(u_{1 \varepsilon}, \ldots, u_{N \varepsilon}\right)\right|},
\end{aligned}
$$

which in light of (3.9) and (3.10) establishes (3.18) upon a time integration.

We finally only need to combine (3.17) with (3.18) to conclude that (3.16) is a consequence of an Aubin-Lions type lemma ([25, Cor. 4]).

Based on this compactness statement, we may conclude the existence of a limit object.

Lemma 3.5. There exist $\left(\varepsilon_{j}\right)_{j \in \mathbb{N}} \subset(0,1)$ and nonnegative functions $u_{1}, \ldots, u_{N}$ defined on $\Omega \times(0, \infty)$ such that $\varepsilon_{j} \searrow 0$ as $j \rightarrow \infty$, and such that for all $i \in\{1, \ldots, N\}$,

$$
u_{i \varepsilon} \rightarrow u_{i} \quad \text { a.e. in } \Omega \times(0, \infty)
$$

and

$$
u_{i \varepsilon} \rightarrow u_{i} \quad \text { in } L_{l o c}^{p}(\bar{\Omega} \times[0, \infty)) \text { for all } p \in\left[1, m_{i}+1\right)
$$

as well as

$$
u_{i \varepsilon} \rightarrow u_{i} \quad \text { in } L_{l o c}^{m_{i}+1}(\bar{\Omega} \times[0, \infty))
$$

as $\varepsilon=\varepsilon_{j} \searrow 0$. 
Proof. In Lemma 3.4 choosing $\zeta=\zeta_{l}$, with $\zeta_{l} \in C_{0}^{\infty}([0, \infty))$ satisfying $\zeta_{l} \equiv 1$ in $[0, l]$ for $l \in \mathbb{N}$, by means of a straightforward extraction procedure relying on the relative compactness of $\left\{u_{i \varepsilon}^{2} \zeta_{l}\left(u_{i \varepsilon}\right) \mid \varepsilon \in(0,1)\right\}$ in $L^{2}(\Omega \times(0, T))$ for any $T>0$ we obtain $\left(\varepsilon_{j}\right)_{j \in \mathbb{N}} \subset(0,1)$ and $u=\left(u_{1}, \ldots, u_{N}\right): \Omega \times(0, \infty) \rightarrow \mathbb{R}^{N}$ such that for all $i \in\{1, \ldots, N\}$ we have $u_{i} \geq 0$ and $u_{i \varepsilon} \rightarrow u_{i}$ a.e. in $\Omega \times(0, \infty)$ as $\varepsilon=\varepsilon_{j} \searrow 0$. Since for each $i \in\{1, \ldots, N\}$ and all $T>0$ we know from Lemma 3.2 that $\left(u_{i \varepsilon}\right)_{\varepsilon \in(0,1)}$ is bounded in $L^{m_{i}+1}(\Omega \times(0, T))$, and that hence $\left(u_{i \varepsilon}^{p}\right)_{\varepsilon \in(0,1)}$ is uniformly integrable over $\Omega \times(0, T)$ for all $p \in\left[1, m_{i}+1\right)$, by reflexivity of $L^{m_{i}+1}(\Omega \times(0, T))$ and the Vitali convergence theorem we readily infer that on passing to a further subsequence if necessary we can also achieve simultaneous validity of (3.21) and (3.22).

Our next goal is to show that the functions just constructed actually form a solution. We begin by confirming that they enjoy a renormalized supersolution property in the style of Definition 2.1. The most crucial ingredient in our verification of this - and actually the reason for dealing with supersolutions - becomes apparent in (3.28), which is enlisted to control the integral involving the gradient $\left(\right.$ of $P_{i}^{(1)}(u)$ ) from above by means of lower semicontinuity.

Lemma 3.6. Let $u_{1}, \ldots, u_{N}$ be as given by Lemma 3.5. Then $u=\left(u_{1}, \ldots, u_{N}\right)$ forms a renormalized supersolution of (1.4) in the sense of Definition 2.1.

Proof. We fix $i \in\{1, \ldots, N\}$ and a nonincreasing convex $\rho \in C^{\infty}([0, \infty))$ such that $\rho^{\prime} \in C_{0}^{\infty}([0, \infty))$, and use (3.1) to see that for all nonnegative $\varphi \in C_{0}^{\infty}(\bar{\Omega} \times[0, \infty))$ such that $\frac{\partial \varphi}{\partial v}=0$ on $\partial \Omega \times(0, \infty)$, we have

$$
\begin{aligned}
& -\int_{0}^{\infty} \int_{\Omega} \rho\left(u_{i \varepsilon}\right) \varphi_{t}-\int_{\Omega} \rho\left(u_{0 i \varepsilon}\right) \varphi(\cdot, 0)=+\int_{0}^{\infty} \int_{\Omega} \partial_{t} \rho\left(u_{i \varepsilon}\right) \varphi \\
& =\int_{0}^{\infty} \int_{\Omega} \rho^{\prime}\left(u_{i \varepsilon}\right) \varphi \cdot\left\{d_{i} m_{i} \nabla \cdot\left(\left(u_{i \varepsilon}+\varepsilon\right)^{m_{i}-1} \nabla u_{i \varepsilon}\right)\right. \\
& \left.+\frac{f_{i}\left(u_{1 \varepsilon}, \ldots, u_{N \varepsilon}\right)}{1+\varepsilon \sum_{j=1}^{N}\left|f_{j}\left(u_{1 \varepsilon}, \ldots, u_{N \varepsilon}\right)\right|}\right\} \\
& =-d_{i} m_{i} \int_{0}^{\infty} \int_{\Omega}\left(u_{i \varepsilon}+\varepsilon\right)^{m_{i}-1} \rho^{\prime \prime}\left(u_{i \varepsilon}\right)\left|\nabla u_{i \varepsilon}\right|^{2} \varphi \\
& -d_{i} m_{i} \int_{0}^{\infty} \int_{\Omega}\left(u_{i \varepsilon}+\varepsilon\right)^{m_{i}-1} \rho^{\prime}\left(u_{i \varepsilon}\right) \nabla u_{i \varepsilon} \cdot \nabla \varphi \\
& +\int_{0}^{\infty} \int_{\Omega} \rho^{\prime}\left(u_{i \varepsilon}\right) \cdot \frac{f_{i}\left(u_{1 \varepsilon}, \ldots, u_{N \varepsilon}\right)}{1+\varepsilon \sum_{j=1}^{N}\left|f_{j}\left(u_{1 \varepsilon}, \ldots, u_{N \varepsilon}\right)\right|} \cdot \varphi \\
& =-d_{i} m_{i} \int_{0}^{\infty} \int_{\Omega}\left|\nabla P_{i \varepsilon}^{(1)}\left(u_{i \varepsilon}\right)\right|^{2} \varphi+d_{i} m_{i} \int_{0}^{\infty} \int_{\Omega} P_{i \varepsilon}^{(2)}\left(u_{i \varepsilon}\right) \Delta \varphi \\
& +\int_{0}^{\infty} \int_{\Omega} \rho^{\prime}\left(u_{i \varepsilon}\right) \cdot \frac{f_{i}\left(u_{1 \varepsilon}, \ldots, u_{N \varepsilon}\right)}{1+\varepsilon \sum_{j=1}^{N}\left|f_{j}\left(u_{1 \varepsilon}, \ldots, u_{N \varepsilon}\right)\right|} \cdot \varphi \quad \text { for all } \varepsilon \in(0,1) \text {, }
\end{aligned}
$$


where we have set

$$
\begin{aligned}
& P_{i \varepsilon}^{(1)}(s):=\int_{0}^{s}(\sigma+\varepsilon)^{\frac{m_{i}-1}{2}} \sqrt{\rho^{\prime \prime}(\sigma)} d \sigma \\
& \text { and } P_{i \varepsilon}^{(2)}(s):=\int_{0}^{s}(\sigma+\varepsilon)^{m_{i}-1} \rho^{\prime}(\sigma) d \sigma, \quad s \geq 0,
\end{aligned}
$$

for $i \in\{1, \ldots, N\}$ and $\varepsilon \in(0,1)$. Here we note that if we take $M>0$ and $T>0$ large enough fulfilling supp $\rho^{\prime} \subset[0, M]$ and $\operatorname{supp} \varphi \subset \bar{\Omega} \times[0, T]$, then for all $\varepsilon \in(0,1)$,

$$
\begin{gathered}
\int_{0}^{\infty} \int_{\Omega}\left|\sqrt{\varphi} \nabla P_{i \varepsilon}^{(1)}\left(u_{i \varepsilon}\right)\right|^{2} \leq\left\|\rho^{\prime \prime}\right\|_{L^{\infty}((0, \infty))}\|\varphi\|_{L^{\infty}(\Omega \times(0, \infty))} \\
\int_{0}^{T} \int_{\Omega} \chi_{\left\{u_{i \varepsilon} \leq M\right\}}\left(u_{i \varepsilon}+\varepsilon\right)^{m_{i}-1}\left|\nabla u_{i \varepsilon}\right|^{2}
\end{gathered}
$$

whence again employing Lemma 3.3 we infer that with $\left(\varepsilon_{j}\right)_{j \in \mathbb{N}}$ as provided by Lemma 3.5 , we can find a subsequence $\left(\varepsilon_{j_{k}}\right)_{k \in \mathbb{N}}$ such that

$$
\sqrt{\varphi} \nabla P_{i \varepsilon}^{(1)}\left(u_{i \varepsilon}\right) \rightarrow z \quad \text { in } L^{2}\left(\Omega \times(0, \infty) ; \mathbb{R}^{n}\right) \quad \text { as } \varepsilon=\varepsilon_{j_{k}} \searrow 0
$$

for some $z \in L^{2}\left(\Omega \times(0, \infty) ; \mathbb{R}^{n}\right)$. On the other hand, since (3.24) entails that $P_{i \varepsilon}^{(1)} \rightarrow$ $P_{i}^{(1)}$ in $L_{l o c}^{\infty}([0, \infty))$ as $\varepsilon \searrow 0$, with $P_{i}^{(1)}$ given by $(2.1)$, and since moreover

$$
\begin{aligned}
\left|P_{i \varepsilon}^{(1)}(s)\right| & \leq\left\|\rho^{\prime \prime}\right\|_{L^{\infty}((0, \infty))}^{\frac{1}{2}} \int_{0}^{M}(\sigma+\varepsilon)^{\frac{m_{i}-1}{2}} d \sigma \\
& =\left\|\rho^{\prime \prime}\right\|_{L^{\infty}((0, \infty))}^{\frac{1}{2}} \cdot \frac{(M+\varepsilon)^{\frac{m_{i}+1}{2}}-\varepsilon^{\frac{m_{i}+1}{2}}}{\frac{m_{i}+1}{2}} \\
& \leq\left\|\rho^{\prime \prime}\right\|_{L^{\infty}((0, \infty))}^{\frac{1}{2}} \cdot \frac{2(M+1)^{\frac{m_{i}+1}{2}}}{m_{i}+1} \quad \text { for all } s \geq 0 \text { and } \varepsilon \in(0,1),
\end{aligned}
$$

from (3.20) and the dominated convergence theorem it follows that

$$
P_{i \varepsilon}^{(1)}\left(u_{i \varepsilon}\right) \rightarrow P_{i}^{(1)}\left(u_{i}\right) \text { in } L^{1}(\Omega \times(0, T)) \quad \text { as } \varepsilon=\varepsilon_{j} \searrow 0 .
$$

Therefore, a standard argument shows that in (3.25) we must have $z=\sqrt{\varphi} \nabla P_{i}^{(1)}\left(u_{i}\right)$ a.e. in $X_{\delta}=\{\varphi>\delta\}$ for all $\delta>0$ (where (3.27) and (3.25) enable us to pass to the limit in the definition of the weak gradient of $P_{i \varepsilon}^{(1)}\left(u_{i \varepsilon}\right):-\int_{X_{\delta}} P_{i}^{(1)}\left(u_{i}\right) \nabla \psi \leftarrow$ $-\int_{X_{\delta}} \nabla \psi P_{i \varepsilon}^{(1)}\left(u_{i \varepsilon}\right)=\int_{X_{\delta}} \psi \nabla P_{i \varepsilon}^{(1)}\left(u_{i \varepsilon}\right) \rightarrow \int_{X_{\delta}} \psi \frac{z}{\sqrt{\varphi}}$ for each $\left.\psi \in C_{0}^{\infty}\left(X_{\delta}\right)\right)$, and hence actually $\sqrt{\varphi} \nabla P_{i \varepsilon}^{(1)}\left(u_{i \varepsilon}\right) \rightarrow \sqrt{\varphi} \nabla P_{i}^{(1)}\left(u_{i}\right)$ in $L_{l o c}^{2}(\Omega \times(0, \infty))$ as $\varepsilon=\varepsilon_{j_{k}} \searrow 0$, so that by lower semicontinuity of the norm in $L^{2}(\Omega \times(0, \infty))$ with respect to weak convergence,

$$
d_{i} m_{i} \int_{0}^{\infty} \int_{\Omega}\left|\nabla P_{i}^{(1)}\left(u_{i}\right)\right|^{2} \varphi \leq \liminf _{\varepsilon=\varepsilon_{j_{k}} \searrow 0}\left\{d_{i} m_{i} \int_{0}^{\infty} \int_{\Omega}\left|\nabla P_{i \varepsilon}^{(1)}\left(u_{i \varepsilon}\right)\right|^{2} \varphi\right\} .
$$


Next addressing the integrals in (3.23) exclusively containing zero-order expressions with respect to $u_{i \varepsilon}$, we first observe that clearly

$$
|\rho(s)| \leq\left\|\rho^{\prime}\right\|_{L^{\infty}((0, \infty))} \cdot M+|\rho(0)| \quad \text { for all } s \geq 0,
$$

and that furthermore, by (3.24), similarly to (3.26) we can estimate

$$
\begin{aligned}
\left|P_{i \varepsilon}^{(2)}(s)\right| & \leq\left\|\rho^{\prime}\right\|_{L^{\infty}((0, \infty))} \int_{0}^{M}(\sigma+\varepsilon)^{m_{i}-1} d \sigma \\
& \leq\left\|\rho^{\prime}\right\|_{L^{\infty}((0, \infty))} \cdot \frac{(M+1)^{m_{i}}}{m_{i}} \quad \text { for all } s \geq 0 \text { and } \varepsilon \in(0,1) .
\end{aligned}
$$

Therefore, three applications of the dominated convergence theorem on the basis of (3.20) and (3.2) show that if we take $P_{i}^{(2)}$ from (2.1) then

$$
\int_{0}^{\infty} \int_{\Omega} \rho\left(u_{i \varepsilon}\right) \varphi_{t} \rightarrow \int_{0}^{\infty} \int_{\Omega} \rho\left(u_{i}\right) \varphi_{t}
$$

and

$$
\int_{\Omega} \rho\left(u_{0 i \varepsilon}\right) \varphi(\cdot, 0) \rightarrow \int_{\Omega} \rho\left(u_{0 i}\right) \varphi(\cdot, 0)
$$

as well as

$$
d_{i} m_{i} \int_{0}^{\infty} \int_{\Omega} P_{i \varepsilon}^{(2)}\left(u_{i \varepsilon}\right) \Delta \varphi \rightarrow d_{i} m_{i} \int_{0}^{\infty} \int_{\Omega} P_{i}^{(2)}\left(u_{i}\right) \Delta \varphi
$$

as $\varepsilon=\varepsilon_{j} \searrow 0$, the latter because in addition obviously $P_{i \varepsilon}^{(2)} \rightarrow P_{i}^{(2)}$ in $L_{l o c}^{\infty}([0, \infty))$ as $\varepsilon \searrow 0$.

Finally, in the crucial rightmost summand in (3.23) containing the reactive contribution, we once more rewrite $f_{i}=\left|f_{i}\right|-2\left(f_{i}\right)_{\text {- and note that fixing any } \delta>0 \text { such }}$ that $(1+\delta) \beta_{j} \leq m_{j}+1$ for all $j \in\{1, \ldots, N\} \backslash\{i\}$, again relying on (1.5) we can estimate

$$
\begin{aligned}
& \int_{0}^{T} \int_{\Omega}\left|\rho^{\prime}\left(u_{i \varepsilon}\right) \cdot \frac{\left(f_{i}\left(u_{1 \varepsilon}, \ldots, u_{N \varepsilon}\right)\right)_{-}}{1+\varepsilon \sum_{j=1}^{N}\left|f_{j}\left(u_{1 \varepsilon}, \ldots, u_{N \varepsilon}\right)\right|} \cdot \varphi\right|^{1+\delta} \\
& \leq \int_{0}^{T} \int_{\Omega}\left|\rho^{\prime}\left(u_{i \varepsilon}\right)\right|^{1+\delta} \cdot\left\{\phi_{i}\left(u_{i \varepsilon}\right)\left(\sum_{j \neq i} u_{j \varepsilon}^{\beta_{j}}+1\right)\right\}^{1+\delta} \cdot \varphi^{1+\delta} \\
& \leq\left\{\left\|\rho^{\prime}\right\|_{L^{\infty}((0, \infty))} \cdot\left\|\phi_{i}\right\|_{L^{\infty}((0, M))} \cdot\|\varphi\|_{L^{\infty}(\Omega \times(0, \infty))}\right\}^{1+\delta} \\
& \cdot N^{1+\delta} \cdot\left(\sum_{j \neq i} \int_{0}^{T} \int_{\Omega} u_{j \varepsilon}^{(1+\delta) \beta_{j}}+|\Omega| T\right)
\end{aligned}
$$

for all $\varepsilon \in(0,1)$. In view of Lemma 3.2, by positivity of $\delta$ this implies uniform integrability of $\left(\rho^{\prime}\left(u_{i \varepsilon}\right) \cdot \frac{\left(f_{i}\left(u_{1 \varepsilon}, \ldots, u_{N \varepsilon}\right)\right)_{-}}{1+\varepsilon \sum_{j=1}^{N}\left|f_{j}\left(u_{1 \varepsilon}, \ldots, u_{N \varepsilon}\right)\right|} \cdot \varphi\right)_{\varepsilon \in(0,1)}$ over $\Omega \times(0, T)$ and hence 
entails, when combined with (3.20), that

$$
\begin{aligned}
& -2 \int_{0}^{\infty} \int_{\Omega} \rho^{\prime}\left(u_{i \varepsilon}\right) \cdot \frac{\left(f_{i}\left(u_{1 \varepsilon}, \ldots, u_{N \varepsilon}\right)\right)_{-}}{1+\varepsilon \sum_{j=1}^{N}\left|f_{j}\left(u_{1 \varepsilon}, \ldots, u_{N \varepsilon}\right)\right|} \cdot \varphi \\
& \rightarrow-2 \int_{0}^{\infty} \int_{\Omega} \rho^{\prime}\left(u_{i}\right)\left(f_{i}\left(u_{1}, \ldots, u_{N}\right)\right)_{-} \varphi
\end{aligned}
$$

as $\varepsilon=\varepsilon_{j} \searrow 0$. Since apart from that, by nonnegativity of both $-\rho^{\prime}$ and $\varphi$ we can invoke Fatou's lemma to see that, again thanks to (3.20),

$$
\begin{aligned}
& -\int_{0}^{\infty} \int_{\Omega} \rho^{\prime}\left(u_{i}\right)\left|f_{i}\left(u_{1}, \ldots, u_{N}\right)\right| \varphi \\
& \leq \liminf _{\varepsilon=\varepsilon_{j} \searrow 0}\left\{-\int_{0}^{\infty} \int_{\Omega} \rho^{\prime}\left(u_{i \varepsilon}\right) \cdot \frac{\left|f_{i}\left(u_{1 \varepsilon}, \ldots, u_{N \varepsilon}\right)\right|}{1+\varepsilon \sum_{j=1}^{N}\left|f_{j}\left(u_{1 \varepsilon}, \ldots, u_{N \varepsilon}\right)\right|} \cdot \varphi\right\}
\end{aligned}
$$

upon collecting (3.28)-(3.33) we altogether conclude from (3.23) that

$$
\begin{aligned}
d_{i} m_{i} \int_{0}^{\infty} \int_{\Omega}\left|\nabla P_{i}^{(1)}\left(u_{i}\right)\right|^{2} \varphi-\int_{0}^{\infty} \int_{\Omega} \rho^{\prime}\left(u_{i}\right)\left|f_{i}\left(u_{1}, \ldots, u_{N}\right)\right| \varphi \\
\leq \liminf _{\varepsilon=\varepsilon_{j_{k}} \searrow 0}\left\{d_{i} m_{i} \int_{0}^{\infty} \int_{\Omega}\left|\nabla P_{i \varepsilon}^{(1)}\left(u_{i \varepsilon}\right)\right|^{2} \varphi\right. \\
\left.\quad-\int_{0}^{\infty} \int_{\Omega} \rho^{\prime}\left(u_{i \varepsilon}\right) \cdot \frac{\left|f_{i}\left(u_{1 \varepsilon}, \ldots, u_{N \varepsilon}\right)\right|}{1+\varepsilon \sum_{j=1}^{N}\left|f_{j}\left(u_{1 \varepsilon}, \ldots, u_{N \varepsilon}\right)\right|} \cdot \varphi\right\} \\
=\liminf _{\varepsilon=\varepsilon_{j_{k}} \searrow 0}\left\{\int_{0}^{\infty} \int_{\Omega} \rho\left(u_{i \varepsilon}\right) \varphi_{t}+\int_{\Omega} \rho\left(u_{0 i \varepsilon}\right) \varphi(\cdot, 0)\right. \\
\quad+d_{i} m_{i} \int_{0}^{\infty} \int_{\Omega} P_{i \varepsilon}^{(2)}\left(u_{i \varepsilon}\right) \Delta \varphi \\
\left.\quad-2 \int_{0}^{\infty} \int_{\Omega} \rho^{\prime}\left(u_{i \varepsilon}\right) \cdot \frac{\left(f_{i}\left(u_{1 \varepsilon}, \ldots, u_{N \varepsilon}\right)\right)_{-}}{1+\varepsilon \sum_{j=1}^{N}\left|f_{j}\left(u_{1 \varepsilon}, \ldots, u_{N \varepsilon}\right)\right|} \cdot \varphi\right\} \\
=\int_{0}^{\infty} \int_{\Omega} \rho\left(u_{i}\right) \varphi_{t}+\int_{\Omega} \rho\left(u_{0 i}\right) \varphi(\cdot, 0) \\
\quad+d_{i} m_{i} \int_{0}^{\infty} \int_{\Omega} P_{i}^{(2)}\left(u_{i}\right) \Delta \varphi-2 \int_{0}^{\infty} \int_{\Omega} \rho^{\prime}\left(u_{i}\right)\left(f_{i}\left(u_{1}, \ldots, . u_{N}\right)\right)_{-} \varphi,
\end{aligned}
$$

which is equivalent to the desired inequality (2.3). The integrability requirements in (2.2) are evident by-products of the above considerations.

But also the subsolution property encoded in (2.5) is fulfilled:

Lemma 3.7. The function $u=\left(u_{1}, \ldots, u_{N}\right)$ from Lemma 3.5 satisfies (2.4) and (2.5) of Definition 2.2.

Proof. According to (3.21), we can pick a null set $N \subset(0, \infty)$ such that with $\left(\varepsilon_{j}\right)_{j \in \mathbb{N}}$ taken from Lemma 3.5, for each $t \in(0, \infty) \backslash N$ we have $u_{i \varepsilon}(\cdot, t) \rightarrow u_{i}(\cdot, t)$ in $L^{1}(\Omega)$ 
for all $i \in\{1, \ldots, N\}$ and hence

$$
\int_{\Omega} \sum_{i=1}^{N} a_{i} u_{i \varepsilon}(\cdot, t) \rightarrow \int_{\Omega} \sum_{i=1}^{N} a_{i} u_{i}(\cdot, t)
$$

as $\varepsilon=\varepsilon_{j} \searrow 0$, where $a \in(0, \infty)^{N}$ is taken from (1.3). We next let $F_{\varepsilon}\left(s_{1}, \ldots, s_{N}\right):=$ $\sum_{i=1}^{N} \frac{a_{i} f_{i}\left(s_{1}, \ldots, s_{N}\right)}{1+\varepsilon \sum_{j=1}^{N}\left|f_{j}\left(s_{1}, \ldots, s_{N}\right)\right|} \quad$ and $\quad F\left(s_{1}, \ldots, s_{N}\right) \quad:=\quad \sum_{i=1}^{N} a_{i} f_{i}\left(s_{1}, \ldots, s_{N}\right)$ for $\left(s_{1}, \ldots, s_{N}\right) \in \mathbb{R}^{N}$ and $\varepsilon \in(0,1)$, and then obtain from (1.3) that

$$
\left(F_{\varepsilon}\left(u_{1 \varepsilon}, \ldots, u_{N \varepsilon}\right)\right)_{+} \leq K \sum_{i=1}^{N} a_{i} u_{i \varepsilon}+K \quad \text { in } \Omega \times(0, \infty) \quad \text { for all } \varepsilon \in(0,1) .
$$

In view of (3.21) applied to $p:=1$, a version of the dominated convergence theorem thus ensures that

$$
\begin{aligned}
& \int_{0}^{t} \int_{\Omega}\left(F_{\varepsilon}\left(u_{1 \varepsilon}, \ldots, u_{N \varepsilon}\right)\right)_{+} \rightarrow \int_{0}^{t} \int_{\Omega} F_{+}\left(u_{1}, \ldots, u_{N}\right) \\
& \quad \text { for all } t>0 \quad \text { as } \varepsilon=\varepsilon_{j} \searrow 0,
\end{aligned}
$$

because clearly $\left(F_{\varepsilon}\left(u_{1 \varepsilon}, \ldots, u_{N \varepsilon}\right)\right)_{+} \rightarrow F_{+}\left(u_{1}, \ldots, u_{N}\right)$ a.e. in $\Omega \times(0, \infty)$ as $\varepsilon=$ $\varepsilon_{j} \searrow 0$ by (3.20). Since from (3.1) we know that for all $t>0$ and $\varepsilon \in(0,1)$ we have

$$
\begin{aligned}
& \int_{\Omega} \sum_{i=1}^{N} a_{i} u_{i \varepsilon}(\cdot, t)+\int_{0}^{t} \int_{\Omega}\left(F_{\varepsilon}\left(u_{1 \varepsilon}, \ldots, u_{N \varepsilon}\right)\right)_{-}=\int_{\Omega} \sum_{i=1}^{N} a_{i} u_{0 i \varepsilon} \\
& \quad+\int_{0}^{t} \int_{\Omega}\left(F_{\varepsilon}\left(u_{1 \varepsilon}, \ldots, u_{N \varepsilon}\right)\right)_{+},
\end{aligned}
$$

where by (3.20) also $\left(F_{\varepsilon}\left(u_{1 \varepsilon}, \ldots, u_{N \varepsilon}\right)\right)_{-} \rightarrow F_{-}\left(u_{1}, \ldots, u_{N}\right)$ a.e. in $\Omega \times(0, \infty)$ as $\varepsilon=\varepsilon_{j} \searrow 0$, and where $\int_{\Omega} \sum_{i=1}^{N} a_{i} u_{0 i \varepsilon} \rightarrow \int_{\Omega} \sum_{i=1}^{N} a_{i} u_{0 i}$ as $\varepsilon \searrow 0$ due to (3.2), invoking Fatou's lemma we infer by means of (3.34) and (3.35) that

$$
\begin{aligned}
& \int_{\Omega} \sum_{i=1}^{N} a_{i} u_{i}(\cdot, t)+\int_{0}^{t} \int_{\Omega} F_{-}\left(u_{1}, \ldots, u_{N}\right) \leq \int_{\Omega} \sum_{i=1}^{N} a_{i} u_{0 i} \\
& +\int_{0}^{t} \int_{\Omega} F_{+}\left(u_{1}, \ldots, u_{N}\right) \quad \text { for all } t \in(0, \infty) \backslash N .
\end{aligned}
$$

For any such $t$, this firstly implies that $F\left(u_{1}, \ldots, u_{N}\right)$ belongs to $L^{1}(\Omega \times(0, t))$, and secondly entails that (2.5) holds.

The previous two lemmata already demonstrate that $u$ is a generalized solution in the sense of Definition 2.2:

Proof of Theorem 1.1. We take $u_{1}, \ldots, u_{N}$ as given by Lemma 3.5 and then only need to combine Lemma 3.6 with Lemma 3.7. 


\section{Acknowledgements}

The authors warmly thank the anonymous reviewers for their thorough evaluation in all details, and for numerous helpful comments which have led to substantial improvement. Apart from that, the second author acknowledges support of the Deutsche Forschungsgemeinschaft in the context of the project Emergence of structures and advantages in cross-diffusion systems (No. 411007140, GZ: WI 3707/5-1).

\section{Funding Open Access funding enabled and organized by Projekt DEAL.}

Open Access. This article is licensed under a Creative Commons Attribution 4.0 International License, which permits use, sharing, adaptation, distribution and reproduction in any medium or format, as long as you give appropriate credit to the original author(s) and the source, provide a link to the Creative Commons licence, and indicate if changes were made. The images or other third party material in this article are included in the article's Creative Commons licence, unless indicated otherwise in a credit line to the material. If material is not included in the article's Creative Commons licence and your intended use is not permitted by statutory regulation or exceeds the permitted use, you will need to obtain permission directly from the copyright holder. To view a copy of this licence, visit http://creativecommons.org/licenses/ by/4.0/.

Publisher's Note Springer Nature remains neutral with regard to jurisdictional claims in published maps and institutional affiliations.

\section{REFERENCES}

[1] H. Amann. Nonhomogeneous linear and quasilinear elliptic and parabolic boundary value problems. In Function spaces, differential operators and nonlinear analysis (Friedrichroda, 1992), volume 133 of Teubner-Texte Math., pages 9-126. Teubner, Stuttgart, 1993.

[2] J. A. Cañizo, L. Desvillettes, and K. Fellner. Improved duality estimates and applications to reactiondiffusion equations. Comm. Partial Differential Equations, 39(6):1185-1204, 2014.

[3] M. C. Caputo, T. Goudon, and A. F. Vasseur. Solutions of the 4-species quadratic reaction-diffusion system are bounded and $C^{\infty}$-smooth, in any space dimension. Anal. PDE, 12(7):1773-1804, 2019.

[4] M. C. Caputo and A. Vasseur. Global regularity of solutions to systems of reaction-diffusion with sub-quadratic growth in any dimension. Comm. Partial Differential Equations, 34(10-12):12281250, 2009.

[5] B. P. Cupps, J. Morgan, and B. Q. Tang. Uniform boundedness for reaction-diffusion systems with mass dissipation. SIAM J. Math. Anal., 53(1):323-350, 2021.

[6] L. Desvillettes, K. Fellner, M. Pierre, and J. Vovelle. Global existence for quadratic systems of reaction-diffusion. Adv. Nonlinear Stud., 7(3):491-511, 2007.

[7] R. J. DiPerna and P.-L. Lions. On the Cauchy problem for Boltzmann equations: global existence and weak stability. Ann. of Math. (2), 130(2):321-366, 1989.

[8] K. Fellner, E. Latos, and B. Q.Tang. Global regularity and convergence to equilibrium of reactiondiffusion systems with nonlinear diffusion. J. Evol. Equ., 20: 957-1003, 2020.

[9] K. Fellner, J. Morgan, and B. Q. Tang. Global classical solutions to quadratic systems with mass control in arbitrary dimensions. Ann. Inst. H. Poincaré Anal. Non Linéaire, 37(2):281-307, 2020.

[10] J. Fischer. Global existence of renormalized solutions to entropy-dissipating reaction-diffusion systems. Arch. Ration. Mech. Anal., 218(1):553-587, 2015.

[11] D. Gilbarg and N. S. Trudinger. Elliptic partial differential equations of second order. Classics in Mathematics. Springer-Verlag, Berlin, 2001. Reprint of the 1998 edition.

[12] Y. I. Kanel'. Solvability in the large of a system of reaction-diffusion equations with the balance condition. Differentsial' nye Uravneniya, 26(3):448-458, 549, 1990. 
[13] E. H. Laamri. Étude de l'existence de solutions globales d'un système de réaction-diffusion parabolique fortement non linéaire. Ann. Fac. Sci. Toulouse Math. (5), 12(3):373-390, 1991.

[14] E.-H. Laamri and B. Perthame. Reaction-diffusion systems with initial data of low regularity. $J$. Differential Equations, 269(11):9310-9335, 2020.

[15] E. H. Laamri and M. Pierre. Global existence for reaction-diffusion systems with nonlinear diffusion and control of mass. Ann. Inst. H. Poincaré Anal. Non Linéaire, 34(3):571-591, 2017.

[16] O. A. Ladyženskaja, V. A. Solonnikov, and N. N. Ural'ceva. Linear and quasilinear equations of parabolic type. Translated from the Russian by S. Smith. Translations of Mathematical Monographs, Vol. 23. American Mathematical Society, Providence, R.I., 1968.

[17] J. Lankeit and M. Winkler. A generalized solution concept for the Keller-Segel system with logarithmic sensitivity: global solvability for large nonradial data. NoDEA Nonlinear Differential Equations Appl., 24(4):Paper No. 49, 33, 2017.

[18] R. H. Martin and M. Pierre. Influence of mixed boundary conditions in some reaction-diffusion systems. Proc. Roy. Soc. Edinburgh Sect. A, 127(5):1053-1066, 1997.

[19] M. Pierre. Weak solutions and supersolutions in $L^{1}$ for reaction-diffusion systems. J. Evol. Equ. 3 (1):153-168, 2003.

[20] M. Pierre. Global existence in reaction-diffusion systems with control of mass: a survey. Milan J. Math., 78(2):417-455, 2010.

[21] M. Pierre and G. Rolland. Global existence for a class of quadratic reaction-diffusion systems with nonlinear diffusions and $L^{1}$ initial data. Nonlinear Anal., 138:369-387, 2016.

[22] M. Pierre and D. Schmitt. Blowup in reaction-diffusion systems with dissipation of mass. SIAM J. Math. Anal., 28(2):259-269, 1997.

[23] M. Pierre, T. Suzuki, and H. Umakoshi. Global-in-time behavior of weak solutions to reactiondiffusion systems with inhomogeneous Dirichlet boundary condition. Nonlinear Anal., 159:393407, 2017.

[24] P. Quittner and P. Souplet. Superlinear parabolic problems. Birkhäuser Advanced Texts: Basler Lehrbücher. Birkhäuser/Springer, Cham, 2019.

[25] J. Simon. Compact sets in the space $L^{p}(0, T ; B)$. Ann. Mat. Pura Appl. (4), 146:65-96, 1987.

[26] P. Souplet. Global existence for reaction-diffusion systems with dissipation of mass and quadratic growth. J. Evol. Equ., 18(4):1713-1720, 2018.

[27] M. Winkler. Large-data global generalized solutions in a chemotaxis system with tensor-valued sensitivities. SIAM J. Math. Anal., 47(4):3092-3115, 2015.

[28] M. Winkler. Global mass-preserving solutions in a two-dimensional chemotaxis-Stokes system with rotational flux components. J. Evol. Equ., 18(3):1267-1289, 2018.

[29] A. Zhigun. Generalized global supersolutions with mass control for systems with taxis. SIAM J. Math. Anal., 51(3):2425-2443, 2019.

\author{
Johannes Lankeit \\ Institut für Angewandte Mathematik \\ Leibniz Universität Hannover \\ Welfengarten 1 \\ 30167 Hannover \\ Germany \\ E-mail:lankeit@ifam.uni-hannover.de
}

Michael Winkler

Institut für Mathematik

Universität Paderborn

Warburger Str. 100

33098 Paderborn

Germany

E-mail:michael.winkler@math.uni-paderborn.de 